\title{
Pooling Modalities and Pointwise Intersection: Semantics, Expressivity, and Dynamics
}

\author{
Frederik Van De Putte ${ }^{1,2}$ (iD $\cdot$ Dominik Klein ${ }^{3}$
}

Received: 3 June 2019 / Accepted: 17 September 2021 / Published online: 7 January 2022

(C) The Author(s) 2021

\begin{abstract}
We study classical modal logics with pooling modalities, i.e. unary modal operators that allow one to express properties of sets obtained by the pointwise intersection of neighbourhoods. We discuss salient properties of these modalities, situate the logics in the broader area of modal logics (with a particular focus on relational semantics), establish key properties concerning their expressive power, discuss dynamic extensions of these logics and provide reduction axioms for the latter.
\end{abstract}

Keywords Classical modal logics · Pointwise intersection · Pooling modalities · Distributed information · Information dynamics · Power delegation

\section{Introduction}

Neighbourhood models are a well-established tool to study generalizations and variants of relational semantics and non-normal modal logics. ${ }^{1}$ They have been successfully applied to i.a. the dynamics of evidence and beliefs [40], the logic of ability $[8,32]$, conflict-tolerant deontic logic [17], the logical relations between obligations

\footnotetext{
${ }^{1}$ Scott [35] and Montague [29] are often seen as the inventors of neighbourhood models; Chellas [10] and Segerberg [36] are usually cited as the main figures in their early development.

$\bowtie$ Frederik Van De Putte

Vandeputte@esphil.eur.nl

Dominik Klein

D.klein@uu.nl

1 Erasmus Institute for Philosophy and Economics, Erasmus School of Philosophy, Erasmus University of Rotterdam, Rotterdam, Netherlands

2 Centre for Logic and Philosophy of Science, Department of Philosophy and Moral Science, Ghent University (Belgium), Ghent, Belgium

3 Department of Philosophy and Religious Studies, Utrecht University, Utrecht, Netherlands
} 
and goals [24], and the analysis of (descriptive or normative) conditionals [9, 26]. The generalization from relational semantics to neighbourhood semantics allows one to invalidate certain schemata that are problematic for a given interpretation of the modal operator $\square$, but also to include other schemata that would trivialize any normal modal logic. ${ }^{2}$ Apart from giving us more logical options, neighbourhood models can also be used as a purely technical tool in order to prove completeness or incompleteness with respect to other semantics. ${ }^{3}$ Finally, neighbourhood models bear very close links to topological models [27, 39] and to subset spaces [30]. ${ }^{4}$

A neighbourhood collection $\mathcal{N}(w)$ for a unary modality $\square$ generalizes the idea of a set $X$ of accessible worlds (from a given world $w$, by a relation $R$ ) that is familiar from relational semantics to a set of accessible sets $X_{1}, X_{2}, \ldots$ of worlds, called the neighbourhoods of $w$. On one specific interpretation of the modal operator, $\square \varphi$ is true iff some neighbourhood $X$ of $w$ is identical to the truth set of $\varphi .^{5}$ Extensions of propositional classical logic with a modal operator $\square$ of this type are standardly called classical modal logics following [10] and include normal modal logics as a special case.

Just as for relational semantics, one can study classical modal logics that are the result of imposing certain relations on the neighbourhood functions, or of treating some neighbourhood functions as defined from others. In particular, the standard operations of non-deterministic choice, sequential composition, and iteration from PDL [23] have been generalized to various types of neighbourhood semantics and formal languages, cf. [33, 38]. In [20, 21], operations on monotonic neighbourhood models are studied from an abstract, algebraic viewpoint, giving rise to highly generic completeness results. ${ }^{6}$ However, notwithstanding these important achievements, the counterpart of intersections of accessibility relations for neighbourhood semantics is largely unknown.

The current paper fills this gap by introducing and studying the notion of pointwise intersection. Let us explain this concept by means of a simple example - exact details will be provided in subsequent sections. Suppose that $\mathcal{N}_{1}(w)$ and $\mathcal{N}_{2}(w)$ are two neighbourhood collections, representing the beliefs of agent 1 , resp. agent 2 at the world $w$. Let $\mathcal{N}_{1}(w)=\{X, Y\}$ and $\mathcal{N}_{2}(w)=\{Z\}$, where each of $X, Y$, and $Z$ are sets of possible worlds. Then the pointwise intersection of $\mathcal{N}_{1}(w)$ and $\mathcal{N}_{2}(w)$ is defined as the set $\mathcal{X}$ of all sets $V \cap U$, where $V \in \mathcal{N}_{1}(w)$ and $U \in \mathcal{N}_{2}(w)$. In particular,

$$
\mathcal{N}_{1}(w) \cap \mathcal{N}_{2}(w)=\{X \cap Z, Y \cap Z\}
$$

\footnotetext{
${ }^{2}$ Examples are the axioms $\square \perp$ and $\square(\varphi \vee \psi) \rightarrow(\square \varphi \wedge \square \psi)$, which have been studied in the context of deontic reasoning, cf. [41], and the axiom $(\square(\varphi \vee \psi) \wedge \neg \square \varphi) \rightarrow \square \psi$ that is considered for logics of agency, cf. [28].

${ }^{3}$ One prototypical example of a completeness proof via neighbourhood semantics is [26]. In [19], neighbourhood semantics are used to prove the incompleteness of Elgesem's modal logic of agency [12].

${ }^{4}$ We refer to [31] for a critical introduction to the many forms, uses and advantages of neighbourhood semantics.

${ }^{5}$ We refer to Section 2 for all definitions of these concepts. We discuss the alternative, "monotonic" interpretation of $\square$ in Section 4.3.

${ }^{6} \mathrm{~A}$ neighbourhood model is monotonic if and only if at every world, the set of neighbourhoods is closed under supersets. See also Section 2.
} 
Pointwise intersection is however not just limited to binary (or finite) combinations of distinct sets of neighbourhoods: one may also intersect the members of one neighbourhood collection, or use several members of one neighbourhood collection in combination with members of other neighbourhood collections, in forming a new neighbourhood. Continuing with our example, we have:

and

$$
\mathcal{N}_{1}(w) \cap \mathcal{N}_{1}(w)=\{X \cap X, Y \cap Y, X \cap Y\}=\{X, Y, X \cap Y\}
$$

$\mathcal{N}_{1}(w) \cap \mathcal{N}_{1}(w) \cap \mathcal{N}_{2}(w)=\{X \cap X \cap Z, Y \cap Y \cap Z, X \cap Y \cap Z\}=\{X \cap Z, Y \cap Z, X \cap Y \cap Z\}$

More generally, given any function $M$ that specifies, for each neighbourhood function $\mathcal{N}_{i}$ in the original model, how many members of $\mathcal{N}_{i}(w)$ should go in the intersection for the world $w$, we can define a unique new neighbourhood function $\mathcal{N}_{M}$. This new neighbourhood function can then be used to interpret a corresponding classical modal operator $\square_{M}$.

We call operators of the type $\square_{M}$ pooling modalities, as they allow us to express information that would be obtained if certain pieces of information or attitudes whether doxastic, evidential, agentive, deontic, or other - are pooled, i.e. combined by means of intersection.

After introducing pooling modalities and their semantics in general and exact terms, we will establish three claims in this paper. First, the operation of pointwise intersection generalizes the intersection of accessibility relations in the exact same way that neighbourhood models generalize relational models. Second and relatedly, pooling modalities add considerable expressive power to the basic (multi)modal language, allowing us to express various properties of neighbourhood models. Third, relying on this expressive power, pooling modalities allow us to formalize various types of dynamics in a multi-agent setting, such as e.g. sharing evidence with others, or delegating one's powers to some other agent.

In the companion paper [43], we prove that notwithstanding their expressive power, many of the resulting logics are well-behaved, i.e. they have an elegant and unified sound and strongly complete axiomatization, they enjoy the finite model property, and they are decidable. Taken as a whole, these two papers are meant to provide a solid foundation for further research on applications of pooling modalities, their metatheory, and related formal languages. ${ }^{7}$

This paper is structured as follows. In Section 2 we recall some basics of neighbourhood semantics and classical modal logics. Section 3 spells out the exact

\footnotetext{
${ }^{7}$ In [31, Section 3.3], the idea of pointwise intersection and pooling modalities is introduced (using different terms) as a generalization of distributed belief, but no formal results about the ensuing logics are given. In [42], Van De Putte and Klein established completeness results for fragments of some of the logics that are studied in the current paper. The main differences between [42] and the present paper are: (a) [42] only concerns pointwise intersections of distinct neighbourhoods, hence, not of a single neighbourhood with itself or more complex combinations with certain neighbourhoods being used more than once; as a result, (b) [42] does not discuss operations of "arbitrary intersection". Also, (c) in the present paper, we include the universal modality, and (d) consider the monotonic semantic clause (cf. Section 4.3). Finally, (e) [42] is only concerned with axiomatizations and completeness, whereas here we include results on expressivity and dynamic operators, and (in [43]) establish the finite model property and decidability of the logics in question.
} 
definition of pointwise intersection as an operation on neighbourhood models, and introduces various formal languages that feature pooling modalities. In Section 4 we investigate the relation between pooling modalities and intersection in relational semantics in detail, and situate our formalism in the landscape of classical modal logics more generally. The expressive power of formal languages with pooling modalities is discussed in Section 5. In Section 6, finally, we spell out a broad class of update types, illustrate their use for specific applications, and show how the ensuing dynamic operators can be expressed and axiomatized by means of static pooling modalities. Section 7 concludes with a list of open problems.

\section{Neighbourhood Models: a Quick Rehearsal}

To set the stage for our contribution, we recall the basics of neighbourhood semantics, fixing notation and terminology along the way. Readers who are familiar with [31] can skip this section.

Basic Languages We start by introducing two formal languages. Fix a countable set $I=\{1,2, \ldots\}$ of indexes $^{8}$ and a countable set $\mathfrak{P}=\left\{p_{1}, p_{2}, \ldots\right\}$ of propositional variables. Where $p$ ranges over $\mathfrak{P}$ and $i$ over $I$, the language $\mathfrak{L}$ is given by the following Backus-Naur form (BNF):

$$
\varphi:=p|\perp| \neg \varphi|\varphi \vee \varphi| \square_{i} \varphi .
$$

The classical connectives $\wedge, \rightarrow, \leftrightarrow$ and the constant $\top$ are defined according to the well-known classical logic schemata. In the remainder of this paper, $\varphi, \psi, \ldots$ are used as metavariables for formulas, and $\Gamma, \Delta, \ldots$ as metavariables for sets of such formulas. Conjunction $(\bigwedge)$ and disjunction $(\bigvee)$ over finite sets $\Delta$ are defined in the standard way. ${ }^{9}$

For the sake of expressive power and in order to characterize specific frame conditions it is common to extend $\mathfrak{L}$ with a universal modality [ $\forall$ ], cf. [18]. The resulting language $\mathfrak{L}^{[\forall]}$ is given by the BNF:

$$
\varphi:=p|\perp| \neg \varphi|\varphi \vee \varphi| \square_{i} \varphi \mid[\forall] \varphi .
$$

We use $[\exists]$ to denote the dual of $[\forall]$. In the remainder, we refer to $\mathfrak{L}$ and $\mathfrak{L}^{[\forall]}$ as the two basic languages that will be enriched with pooling modalities in Section 3.

Frames and Models A frame $\mathfrak{F}$ is a tuple that specifies a non-empty set of possible worlds $W$ and, for each index $i \in I$ and $w \in W$, a corresponding set $\mathcal{N}_{i}(w)$ of subsets of $W$, called the neighbourhoods of $i$ at $w$. We will use the term neighbourhood set (for $i$ ) to refer to the set $\mathcal{N}_{i}(w)$. Depending on the application, $\mathcal{N}_{i}(w)$ may e.g. denote the beliefs that agent $i$ holds at $w$, the permissions that are granted in view of

\footnotetext{
${ }^{8}$ Depending on the specific application, indexes may be interpreted in various ways: they may refer to agents whose beliefs, evidence, or abilities we want to model; to legal or moral codes implying certain obligations or permissions; or to resources enabling a given agent to guarantee certain states of affairs.

${ }^{9}$ If $\Delta=\emptyset$, then $\bigvee \Delta=_{\text {df }} \top$ and $\bigwedge \Delta=$ df $\perp$.
} 
normative system $i$ at $w$, or the propositions one can guarantee given the resource $i$ at $w$.

To interpret $\mathfrak{L}^{[\forall]}$, we extend frames to models (Definition 1) and give semantic clauses for each of the components of $\mathfrak{L}^{[\forall]}$ (Definition 2):

Definition 1 A model $\mathfrak{M}$ is a triple $\left\langle W,\left\langle\mathcal{N}_{i}\right\rangle_{i \in I}, V\right\rangle$, where (i) $W \neq \emptyset$ is the domain of $\mathfrak{M}$, (ii) for every $i \in I, \mathcal{N}_{i}: W \rightarrow \wp(\wp(W))$ is a neighbourhood function for $i$, and (iii) $V: \mathfrak{P} \rightarrow \wp(W)$ is a valuation function.

Definition 2 Where $\mathfrak{M}=\left\langle W,\left\langle\mathcal{N}_{i}\right\rangle_{i \in I}, V\right\rangle$ is a model and $w \in W$ :

0. $\mathfrak{M}, w \not \models \perp$

1. $\mathfrak{M}, w \models \varphi$ iff $w \in V(\varphi)$ for all $\varphi \in \mathfrak{P}$

2. $\mathfrak{M}, w \models \neg \varphi$ iff $\mathfrak{M}, w \notin \varphi$

3. $\mathfrak{M}, w \models \varphi \vee \psi$ iff $\mathfrak{M}, w \models \varphi$ or $\mathfrak{M}, w \models \psi$

4. $\mathfrak{M}, w \models \square_{i} \varphi$ iff $\|\varphi\|^{\mathfrak{M}} \in \mathcal{N}_{i}(w)$

5. $\mathfrak{M}, w \models[\forall] \varphi$ iff $\mathfrak{M}, w^{\prime} \models \varphi$ for all $w^{\prime} \in W$.

where $\|\varphi\|^{\mathfrak{M}}=\{w \in W \mid \mathfrak{M}, w \models \varphi\}$.

Validity $(\Vdash \varphi)$ and semantic consequence $(\Gamma \Vdash \varphi)$ are defined in the standard way, viz. as truth, resp. truth-preservation at all worlds in all models.

The semantics just presented captures the so-called exact reading of the modal operators $\square_{i}$ : for $\square_{i} \varphi$ to be true at $w$, the exact truth set of $\varphi$ has to be in the neighbourhood of $i$ at $w$. This is often contrasted with a weaker reading where $\square_{i} \varphi$ is true iff some neighbourhood of $i$ at $w$ is a (perhaps proper) subset of $\|\varphi\|^{\mathfrak{M}}$. We will discuss this weaker reading in more detail in Section 4.3.

Example 1 Consider a model $\mathfrak{M}=\left\langle W,\left\langle\mathcal{N}_{i}\right\rangle_{i \in I}, V\right\rangle$ with $W=\{w, v, u\}, \mathcal{N}_{1}(w)=$ $\{w, v\},\{v, u\}$, and $\mathcal{N}_{2}(w)=\{w, u\}$, and $V(p)=\{w, v\}, V(q)=\{v, u\}$. In this model, $\square_{1} p, \square_{1} q$, and $\square_{2}(\neg p \vee \neg q)$ are all true at $w,[\forall](p \vee q)$ is true at each world. Figure 1 gives a graphic representation of this model.

Frame Conditions As the above example illustrates, the semantics and logic characterized by the class of all models is very weak. Note that $\square_{1}(p \wedge q), \square_{1}(p \vee q)$, and $\square_{1} \top$ are false at $w$ in this model. These facts illustrate the failure of three wellknown properties of logics characterized by relational semantics, viz. aggregation, monotony, and necessitation. ${ }^{10}$ For $\mathfrak{L}$, the class of all neighbourhood models gives us the weakest modal logic that satisfies the principle of replacement of equivalents (RE):

$$
\text { if } \Vdash \varphi \leftrightarrow \psi \text {, then } \square_{i} \varphi \rightarrow \square_{i} \psi
$$

\footnotetext{
${ }^{10}$ See Table 1 where those properties are specified.
} 


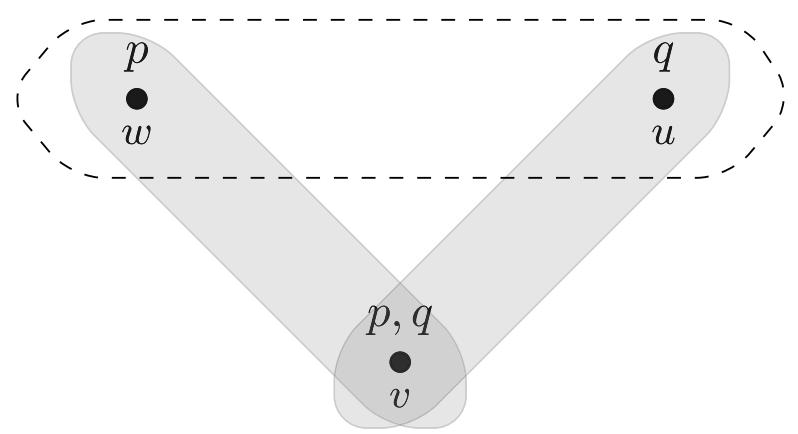

Fig. 1 A representation of the model in Example 1. $\mathcal{N}_{1}(w)$ consists of the grey sets, $\mathcal{N}_{2}(w)$ of the dashed set

To characterize the weakest logic in $\mathfrak{L}^{[\forall]}$, we need to add all axioms of $\mathbf{S 5}$ for $[\forall]$ and replace (RE) with the axiom of replacement of global equivalents (RGE): ${ }^{11}$

$$
[\forall](\varphi \leftrightarrow \psi) \rightarrow\left(\square_{i} \varphi \rightarrow \square_{i} \psi\right)
$$

Having applications to doxastic logic, deontic logic, or the logic of ability in mind, one may require various additional properties of the logic. Such properties are to be had if we impose additional frame conditions, i.e., conditions on the frames underlying our models. Frame conditions can also be motivated in terms of the interpretation of the models. For instance, if we interpret the members of a neighbourhood as "those states of affairs the agent can guarantee, independently of any other agent", then it is common to require the neighbourhood sets to be closed under supersets.

Table 1 lists some standard axiom schemata and frame conditions that characterize them. Here and in the remainder, $\mathcal{X}^{\uparrow}$ denotes the closure of $\mathcal{X}$ under supersets. ${ }^{12}$ The quantification over $w$ in the conditions on the neighbourhood functions is universal; e.g. the condition for $(\mathrm{N})$ reads that for all $w \in W, W \in \mathcal{N}_{i}(w)$. Note that, for the last condition in this table, we need the universal modality in order to obtain a characteristic axiom. When some operator $\square_{i}$ satisfies $\left(\mathbf{M}_{i}\right)$, we say it is monotonic.

Since we take a unifying, universal logic perspective on pointwise intersection in this paper, we will not assume the satisfaction of any of the mentioned conditions unless stated otherwise. In particular, all results from this paper apply to arbitrary models of the type given in Definition 1, again, unless stated otherwise.

Augmented models, supplementation As mentioned in the introduction, neighbourhood models are a generalization of relational models for modal logic. More specifically, if we use the standard semantic clause for $\square$ for relational models, and the exact semantic clause for neighbourhood models, then the class of

\footnotetext{
${ }^{11}$ Note that, by necessitation for $[\forall], \Vdash \varphi \leftrightarrow \psi$ entails $\Vdash[\forall](\varphi \leftrightarrow \psi)$. So from $\Vdash \varphi \leftrightarrow \psi$ we can derive $\Vdash \square_{i} \varphi \leftrightarrow \square_{i} \psi$ using (RGE).

${ }^{12}$ Formally, for every $\mathcal{X} \subseteq \wp(D)$ for some domain $D: \mathcal{X}^{\uparrow}=\{Y \subseteq D \mid X \subseteq Y$ for some $X \in \mathcal{X}\}$.
} 
Table 1 Modal axioms and corresponding frame conditions

\begin{tabular}{lll}
\hline$\left(\mathrm{N}_{i}\right)$ & $\square_{i} \top$ & $W \in \mathcal{N}_{i}(w)$ \\
$\left(\mathrm{P}_{i}\right)$ & $\neg \square_{i} \perp$ & $\emptyset \notin \mathcal{N}_{i}(w)$ \\
$\left(\mathrm{T}_{i}\right)$ & $\square_{i} \varphi \rightarrow \varphi$ & for all $X \in \mathcal{N}_{i}(w), w \in X$ \\
$\left(\mathrm{M}_{i}\right)$ & $\square_{i}(\varphi \wedge \psi) \rightarrow\left(\square_{i} \varphi \wedge \square_{i} \psi\right)$ & $\mathcal{N}_{i}(w)=\left(\mathcal{N}_{i}(w)\right)^{\uparrow}$ \\
$\left(\mathrm{C}_{i}\right)$ & $\left(\square_{i} \varphi \wedge \square_{i} \psi\right) \rightarrow \square_{i}(\varphi \wedge \psi)$ & $\mathcal{N}_{i}(w)$ is closed under finite intersections \\
$\left(\mathrm{U}_{i}\right)$ & $\square_{i} \varphi \rightarrow[\forall] \square_{i} \varphi$ & $\mathcal{N}_{i}(w)=\mathcal{N}_{i}\left(w^{\prime}\right)$ for all $w, w^{\prime} \in W$ \\
\hline
\end{tabular}

relational models is modally equivalent to the class of neighbourhood models that are "augmented". ${ }^{13}$ In the present context, the latter notion can be defined as follows:

Definition 3 Let $D$ be a set and $\mathcal{X} \subseteq \wp(D) . \mathcal{X}$ is augmented iff (i) $\mathcal{X}=\mathcal{X}^{\uparrow}$ (i.e., $\mathcal{X}$ is closed under supersets), (ii) $W \in \mathcal{X}$, and (iii) $\mathcal{X}=\cap^{\infty} \mathcal{X}$ (i.e., $\mathcal{X}$ is closed under arbitrary intersections). A model $\mathfrak{M}=\left\langle W,\left\langle\mathcal{N}_{i}\right\rangle_{i \in I}, V\right\rangle$ is augmented iff for all $w \in W$ and all $i \in I, \mathcal{N}_{i}(w)$ is augmented.

A model is called monotonic iff for all $i \in I$, for all $w \in W, \mathcal{N}_{i}(w)$ is closed under supersets. The following well-known notion is useful in the study of monotonic models:

Definition 4 Let $\mathfrak{M}=\left\langle W,\left\langle\mathcal{N}_{i}\right\rangle_{i \in I}, V\right\rangle$ be a model. The supplementation of $\mathfrak{M}$, $\mathfrak{M}^{\uparrow}$, is the triple $\left\langle W,\left\langle\mathcal{N}_{i}^{\uparrow}\right\rangle_{i \in I}, V\right\rangle$, where, for all $w \in W, \mathcal{N}_{i}^{\uparrow}(w)$ is the closure of $\mathcal{N}_{i}(w)$ under supersets.

\section{Pointwise Intersection and Pooling Modalities}

In this section, we enrich the basic languages with pooling modalities. In order to interpret them, we need to introduce the notion of pointwise intersection in full generality. After that, we will discuss some key validities and invalidities of the resulting logics.

Semantics of Pooling Modalities Consider again Example 1 as depicted in Fig. 1. Here, the neighbourhood for index 1 contains $\|p\|$ and $\|q\|$, and the neighbourhood for index 2 contains $\|\neg(p \wedge q)\|$. Suppose we interpret $\square_{i} \varphi$ as " $i$ has some piece of evidence that indicates that $\varphi$ is the case". Under this reading, aggregation is not valid: having pieces of evidence, each for various propositions, does not entail having a single piece of evidence for the conjunction of those propositions. However, in the example, there is some sense in which agent 1 has information that contradicts the information of agent 2. That is, if we would combine the various propositions

\footnotetext{
${ }^{13}$ This result dates back to [10]; see [31, Theorem 2.21] for a recent proof and discussion with the notation we use.
} 
for which 1 has evidence, then we would obtain information that contradicts 2's evidence. Claims like these can be made exact by introducing the notion of pointwise intersection, and expressed in a formal language by pooling modalities.

Let us start with the former:

Definition 5 Let $D$ be a set, let $\mathcal{X}, \mathcal{Y} \subseteq \wp(D)$, and let $k \in \mathbb{N}$.

1. $\mathcal{X} \cap \mathcal{Y}=\{X \cap Y \mid X \in \mathcal{X}, Y \in \mathcal{Y}\}$ is the pointwise intersection of $\mathcal{X}$ and $\mathcal{Y}$.

2. $\cap^{k} \mathcal{X}=\left\{X_{1} \cap \ldots \cap X_{k} \mid X_{1}, \ldots, X_{k} \in \mathcal{X}\right\}$ is the pointwise $k$-intersection of $\mathcal{X}$ with itself.

3. $\Pi^{\infty} \mathcal{X}=\{\bigcap \mathcal{Y} \mid \mathcal{Y} \subseteq \mathcal{X}\}$ is the pointwise arbitrary intersection of $\mathcal{X}$ with itself.

In order to talk about pointwise intersections of neighbourhood sets, we first need a means to denote the indexes $i \in I$ that are being combined, and the number of neighbourhoods in each such $\mathcal{N}_{i}(w)$ that go into the intersection. This requires some additional notation.

Definition 6 Pooling profiles are functions of the type $M: I \rightarrow \mathbb{N} \cup\{\infty\}$, where (i) for only finitely many $i \in I, M(i) \neq 0$ and (ii) for at least one $i \in I, M(i)>0$.

$\mathbb{M}_{\infty}$ denotes the set of all pooling profiles. $\mathbb{M}_{f} \subset \mathbb{M}_{\infty}$ is the set of all finitary pooling profiles, i.e. pooling profiles of the type $M: I \rightarrow \mathbb{N}$.

For every $M \in \mathbb{M}_{\infty}, \mathbf{I}(\mathbf{M})=_{\mathrm{df}}\{i \in I \mid M(i) \neq 0\}$.

When writing about pooling profiles, we will often switch from functional to a simplified relational notation, writing every pooling profile as a finite set of pairs $(i, k)$ for $k \in \mathbb{N}^{+} \cup\{\infty\}$, thus omitting all pairs $(j, 0)$. Note that with such notation, every pooling profile is a finite set.

Intuitively, a pooling profile $M$ indicates, for each $i \in I$, the number of sets $X \in \mathcal{N}_{i}(w)$ that we can use in order to obtain a member $Y$ of the neighbourhood set $\mathcal{N}_{M}(w)$. The symbol $\infty$ can be read as "arbitrarily many". So e.g., that $X \in \mathcal{N}_{\{(1, \infty)\}}(w)$ means that $X$ is the result of intersecting an arbitrary, possibly infinite or even uncountable number of members of $\mathcal{N}_{1}(w)$; that $Y \in \mathcal{N}_{\{(1,2),(2, \infty)\}}(w)$ means that $Y$ can be obtained by intersecting 2 members of $\mathcal{N}_{1}(w)$ with an arbitrary number of members of $\mathcal{N}_{2}(w) .{ }^{14}$

Definition 7 Let $\mathfrak{M}=\left\langle W,\left\langle\mathcal{N}_{i}\right\rangle_{i \in I}, V\right\rangle$ be a neighbourhood model and let $M \in \mathbb{M}_{\infty}$, with $I(M)=\left\{i_{1}, \ldots, i_{n}\right\}$. The neighbourhood function $\mathcal{N}_{M}$ is defined as follows: for every $w \in W$,

$$
\mathcal{N}_{M}(w)=\left(\cap^{M\left(i_{1}\right)} \mathcal{N}_{i_{1}}(w)\right) \cap \ldots \cap\left(\cap^{M\left(i_{n}\right)} \mathcal{N}_{i_{n}}(w)\right)
$$

Since we will often have to refer to specific (relatively small) pooling profiles in examples, we will use a simplified "multiset" notation for them: e.g. $\left\{1,1,2^{\infty}, 3\right\}$

\footnotetext{
14“Arbitrary" should thus be interpreted here in the strongest possible sense, corresponding to the third item of Definition 5.
} 
Table 2 Languages with pooling modalities

\begin{tabular}{lll}
\hline $\mathfrak{L}_{\infty}$ & $\varphi:=p|\perp| \neg \varphi|\varphi \vee \varphi| \square_{M} \varphi$ & \\
$\mathfrak{L}_{\infty}^{[\forall]}$ & $\varphi:=p|\perp| \neg \varphi|\varphi \vee \varphi| \square_{M} \varphi \mid[\forall] \varphi$ & where $p \in \mathfrak{P}$ and $M \in \mathbb{M}_{\infty}$ \\
$\mathfrak{L}_{f}$ & $\varphi:=p|\perp| \neg \varphi|\varphi \vee \varphi| \square_{M} \varphi$ & where $p \in \mathfrak{P}$ and $M \in \mathbb{M}_{\infty}$ \\
$\mathfrak{L}_{f}^{[\forall]}$ & $\varphi:=p|\perp| \neg \varphi|\varphi \vee \varphi| \square_{M} \varphi \mid[\forall] \varphi$ & where $p \in \mathfrak{P}$ and $M \in \mathbb{M}_{f}$ \\
\hline
\end{tabular}

denotes the pooling profile $M$ for which $M(1)=2, M(2)=\infty, M(3)=1$, and $M(i)=0$ for all $i \in I \backslash\{1,2,3\}$. When such sets occur in subscripts we will also omit set brackets whenever this causes no confusion.

Example 2 Consider again Example 1 from the previous section (Fig. 1). In this model, $\{w\} \in \mathcal{N}_{1,2}(w),\{v\} \in \mathcal{N}_{1,1}(w)$, and $\emptyset \in \mathcal{N}_{1,1,2}(w)$.

We are now in a position to define the logics that play central stage in this paper. The formal languages $\mathfrak{L}_{\infty}$ and $\mathfrak{L}_{\infty}^{[\forall]}$ are obtained by replacing $\square_{i}(i \in I)$ with $\square_{M}$ $\left(M \in \mathbb{M}_{\infty}\right)$ in $\mathfrak{L}$, resp. $\mathfrak{L}^{[\forall]}$. In a similar fashion we obtain $\mathfrak{L}_{f}$ and $\mathfrak{L}_{f}^{[\forall]}$, where instead of $\mathbb{M}_{\infty}$ we use $\mathbb{M}_{f}$. Table 2 gives the BNFs of each of these languages.

These languages are interpreted in the exact same way as before (cf. Definition 2), except that we use the following semantic clause for the pooling modalities $\square_{M}$ :

Definition 8 Where $\mathfrak{M}=\left\langle W,\left\langle\mathcal{N}_{i}\right\rangle_{i \in I}, V\right\rangle$ is a neighbourhood model, $M \in \mathbb{M}_{\infty}$, and $w \in W$,

$$
\mathfrak{M}, w \models \square_{M} \varphi \text { iff }\|\varphi\|^{\mathfrak{M}} \in \mathcal{N}_{M}(w) .
$$

Returning to Example 1, we have that $\mathfrak{M}, w \models \square_{1,2}(p \wedge \neg q)$, $\mathfrak{M}, w \models \square_{1,1}(p \wedge q), \mathfrak{M}, w \models \square_{1,1,2} \perp$, and $\mathfrak{M}, w \models \square_{1 \infty, 2} \perp$. The latter formula expresses that there is conflicting information among the group of agents $\{1,2\}$. In contrast, the evidence of agent 1 alone is consistent, and $\mathfrak{M}, w \models \neg \square_{1} \infty \perp$.

It can be easily observed that for the border case where a pooling profile $M$ coincides with a single index $i$, i.e. $M=(i, 1)$, this semantic clause is conservative with respect to the original semantic clause for $\square_{i}$ :

Fact 1 Let $M \in \mathbb{M}_{\infty}$ and $i \in I$ be such that $I(M)=i$ and $M(i)=1$. Then, for all models $\mathfrak{M}=\left\langle W,\left\langle\mathcal{N}_{i}\right\rangle_{i \in I}, V\right\rangle$ and all $w \in W: \mathfrak{M}, w \models \square_{M} \varphi$ iff $\mathfrak{M}, w \models \square_{i} \varphi$.

In view of this fact, the languages with pooling modalities are a conservative extension of the corresponding languages with only single-indexed operators.

Logical Properties of Pooling Modalities To illustrate the above notions and give the reader a feeling of the behavior of pooling modalities, let us go over a few validities and invalidities of this semantics. First, given the well-known correspondence between intersecting sets of worlds and classical conjunction, pooling distinct propositions - whether across different indexes, or within a single index - gives us the 
conjunction of those propositions for the associated pooling profile. The following three validities illustrate this:

$$
\begin{gathered}
\Vdash\left(\square_{1} p \wedge \square_{2} q\right) \rightarrow \square_{1,2}(p \wedge q) \\
\Vdash\left(\square_{1} p \wedge \square_{1} r\right) \rightarrow \square_{1,1}(p \wedge r) \\
\Vdash\left(\square_{1} p \wedge \square_{2} q \wedge \square_{1} r\right) \rightarrow \square_{1,1,2}(p \wedge q \wedge r)
\end{gathered}
$$

Note that in the consequents of Eqs. 2 and 3, the multiset notation allows us to represent the sources of pooled information in a very perspicuous way. Conversely, one may ask whether the pooled information that $\varphi$ given a pooling profile $M$ always entails that $\varphi$ is already attributable to some index $i \in I(M)$. This is not the case: it may be that only by using information that is distributed among the various indexes, or by combining distinct neighbourhoods for a single index, we can arrive at $\varphi$. So e.g.

$$
\begin{gathered}
\| \square_{1,2} p \rightarrow\left(\square_{1} p \vee \square_{2} p\right) \\
\forall \square_{1,1} p \rightarrow \square_{1} p
\end{gathered}
$$

The invalidity of the implication in Eq. 4 is well-known from the literature on intersection modalities in relational semantics, cf. [13]. Equation 5 shows that, in the context of neighbourhood models (since neighbourhoods need not be closed under intersection), the implication also fails in the special case with only one index.

One may also consider the converse of Eq. 4. According to the standard account of distributed belief on relational models, whenever $\varphi$ is believed by some $G$, then $\varphi$ is also distributed belief for every supergroup $H \supset G$. Crucially, the analogue of this implication fails for the weaker, non-normal notions of pooled information that we study here: ${ }^{15}$

$$
\forall \square_{1} p \rightarrow \square_{1,2} p
$$

To see why the implication in Eq. 6 is invalid, consider a model $\mathfrak{M}$ with world $w$ in which the neighbourhood for index 2 is empty, i.e., $\mathcal{N}_{2}=\emptyset$, yet $\|p\| \neq \emptyset$. Then by Definition 7, also $\mathcal{N}_{1,2}(w)=\emptyset$ and hence there is no $\varphi$ such that $\square_{1,2} \varphi$ holds at $w$ in $\mathfrak{M}$. So here already we see that, by abandoning the assumption that every agent must have at least some belief, the associated modalities for distributed belief behave differently. But even if we assume non-emptiness of the neighbourhoods, the implication in Eq. 6 may still fail. That is, suppose that $\mathcal{N}_{1}(w)=\left\{\|p\|^{\mathfrak{M}}\right\}$ and $\mathcal{N}_{2}(w)=\left\{\|q\|^{\mathfrak{M}}\right\}$. Then the only element of $\mathcal{N}_{1,2}(w)$ is $\|p \wedge q\|^{\mathfrak{M}}$, which may be distinct from both $\|p\|$ and $\|q\|$. So in such a case, $\square_{1} p$ holds whereas $\square_{1,2} p$ fails.

More restricted versions of the implication in Eq. 6 do however obtain. First, if we just "enlarge" a pooling profile by adding more occurrences of a given index $i \in I(M)$, then we obtain at least as much pooled information:

$$
\Vdash \square_{1,2}(p \vee r) \rightarrow \square_{1,1,2}(p \vee r)
$$

\footnotetext{
${ }^{15}$ A natural situation where this implication fails is in logics of strong permission or so-called deontic sufficiency [41]. There, $X \in \mathcal{N}_{i}(w)$ can be taken to express that, in order to conform to normative code $i$, it suffices to ensure that $X$ is the case. Accordingly, $\square_{i} \varphi$ means that it suffices to make $\varphi$ true, in order to conform to code $i$. The invalidity of Eq. 6 then amounts to the observation that conforming to one normative code does not imply conforming to that code in combination with some other code.
} 
This follows immediately from the fact that, in our definition of pointwise intersection, we do not require the intersected sets to be distinct. So e.g. where $X \in \mathcal{N}_{1}(w)$ and $Y \in \mathcal{N}_{2}(w)$, we have $X \cap X \cap Y=X \cap Y \in \mathcal{N}_{1,1,2}(w)$.

Second, in case we add a new index $i$, for which we know that $\mathcal{N}_{i}(w)$ contains some proposition $\varphi$ that includes the original proposition $\psi$, then $\psi$ is preserved when moving to the larger pooling profile. In other words, it suffices for that $i$ to have some (possibly redundant) information that includes all $\varphi$-worlds, in order to ensure that the group including $i$ also has the pooled information that $\psi$. Examples of associated validities are:

$$
\Vdash\left(\square_{1} p \wedge \square_{2} \top\right) \rightarrow \square_{1,2} p
$$

and

$$
\Vdash\left(\square_{1,1,2}(\neg p \wedge q) \wedge \square_{3 q}\right) \rightarrow \square_{1,1,2,3}(\neg p \wedge q)
$$

Finally, note that entire domain $W$ of a model can only be obtained by intersecting some sets $X_{1}, \ldots, X_{n}$ when those $X_{i}$ are themselves equal to $W$. As an immediate result, we obtain the following restricted variants of Eq. 4, resp. Eq. 5:

$$
\begin{aligned}
& \Vdash \square_{1,2} \top \rightarrow \square_{1} \top \\
& \Vdash \square_{1,1} \top \rightarrow \square_{1} \top
\end{aligned}
$$

This completes our survey of some salient properties of pooling modalities. Sound and complete axiomatizations of all validities in the respective languages, relative to various combinations of frame conditions, are provided in the companion paper [43].

\section{Relational Semantics as a Special Case}

In this section, we show that pointwise intersection of neighbourhood functions is a generalization of the intersection of accessibility relations. In fact, there are two ways in which normal modal logics with intersection modalities can be captured in terms of pooling modalities:

(a) Over augmented neighbourhood models, pointwise intersection corresponds exactly to the intersection of the accessibility relations in the corresponding relational model (Section 4.1).

(b) Normal modal logics with intersection modalities are a fragment of the pooling logics characterized by the class of monotonic models (Section 4.2).

Claim (a) was already mentioned in [31] for languages without a universal modality; there, the proof is left to the reader. ${ }^{16}$ Claim (b) is new. Whereas neither of these claims come totally unexpected, it is useful to spell them out in exact detail, also for subsequent sections.

In Section 4.3 we consider the monotonic semantic clause for the modal operators $\square_{M}$ and show that, using this clause, we obtain a logic that is equivalent to the logic of monotonic models - thus generalizing a well-known result for mono-modal neighbourhood semantics.

\footnotetext{
${ }^{16}$ See [31, Section 3.3, Exercise 73].
} 


\subsection{Augmented Neighbourhood Models}

Let $\mathbb{G}$ denote the set of all finite, non-empty subsets of $I$. In the remainder we will slightly abuse notation, using $G$ as a variable for both, sets of agents, and pooling profiles, where in the latter case we stipulate that $G=\{(i, 1) \mid i \in G\} \cup\{(j, 0) \mid$ $j \notin G\}$. We focus here on the formal language $\mathfrak{L}_{G}^{[\forall]}$ obtained by adding $[\forall]$ and all operators $\square_{G}$ for $G \in \mathbb{G}$ to classical propositional logic. Analogous observations can be made for the language without these operators.

A first thing to note is that, when interpreted over augmented neighbourhood models, $\mathfrak{L}_{G}^{[\forall]}$ is as expressive as the full language $\mathfrak{L}_{\infty}^{[\forall]}$. That is, since the neighbourhoods of an augmented model are already closed under arbitrary intersection, all distinctions between a given pooling profile $M$ and the underlying set of indexes $I(M)$ are lost:

Fact 2 Let $\mathfrak{M}$ be an augmented neighbourhood model and let $w$ be a member of the domain of $\mathfrak{M}$. Then, for all $M \in \mathbb{M}_{\infty}$ and $\varphi \in \mathfrak{L}_{\infty}^{[\forall]}$, the following holds: $\mathfrak{M}, w \models \square_{M} \varphi$ iff $\mathfrak{M}, w \models \square_{I(M)} \varphi$.

Recall that a relational model $\mathfrak{S}$ is just like a neighbourhood model, with each $\mathcal{N}_{i}$ replaced by a relation $\mathcal{R}_{i} \subseteq W \times W$. The semantic clauses for $\mathfrak{L}_{G}^{[\forall]}$, interpreted over relational models, are the same as for neighbourhood models, except for the modal operators of the type $\square_{G}$ :

Definition 9 Let $\mathfrak{S}=\left\langle W,\left\langle\mathcal{R}_{i}\right\rangle_{i \in I}, V\right\rangle$ be a relational model. For every $G \in \mathbb{G}$, let $\mathcal{R}_{G}=\bigcap_{i \in G} \mathcal{R}_{i}$. For every $w \in W$ and $G \in \mathbb{G}$ :

$$
\mathfrak{S}, w \models \square_{G} \varphi \text { iff } \mathfrak{S}, w^{\prime} \models \varphi \text { for all } w^{\prime} \in \mathcal{R}_{G}(w) .
$$

In order to establish a correspondence between augmented neighbourhood models and relational models, we define transformations from one to the other. ${ }^{17}$ Where $\mathcal{N}: W \rightarrow \wp(\wp(W))$ is a neighbourhood function, let $\mathcal{R}_{\mathcal{N}} \subseteq W \times W$ be defined by putting $\mathcal{R}_{\mathcal{N}}(w)=\bigcap \mathcal{N}(w)$ for all $w \in W$. Conversely, for any accessibility relation $\mathcal{R} \subseteq W \times W$, let $\mathcal{N}_{\mathcal{R}}(w)=\{X \subseteq W \mid \mathcal{R}(w) \subseteq X\}$. In the remainder, we will use the term standard transformations to refer to both transformations. Where $\mathfrak{M}$ is a neighbourhood model, we use $\mathfrak{S}_{\mathfrak{M}}$ to denote the relational model that is obtained from $\mathfrak{M}$ using the standard transformation; conversely, $\mathfrak{M}_{\mathfrak{S}}$ denotes the (augmented) neighbourhood model obtained from $\mathfrak{S}$ by the standard transformation. We now show that the standard transformations preserve validity in the richer languages that feature pooling modalities.

Lemma 3 Let $\mathfrak{S}=\left\langle W,\left\langle\mathcal{R}_{i}\right\rangle_{i \in I}, V\right\rangle$ be a relational model. Then, for all $w \in W$ and $G \in \mathbb{G}$,

$$
\cap_{i \in G} \mathcal{N}_{\mathcal{R}_{i}}(w)=\left\{X \subseteq W \mid \mathcal{R}_{G}(w) \subseteq X\right\}
$$

\footnotetext{
${ }^{17}$ This transformation dates back at least to [10, Theorem 7.9].
} 
Proof $(\subseteq)$ Suppose $X \in \cap_{i \in G} \mathcal{N}_{\mathcal{R}_{i}}(w)$. So there is a set $\left\{Y_{i} \mid i \in G, Y_{i} \in \mathcal{N}_{\mathcal{R}_{i}}(w)\right\}$ such that $\bigcap_{i \in G} Y_{i}=X$. Since each $\mathcal{N}_{\mathcal{R}_{i}}$ is obtained from $\mathcal{R}_{i}$ by the standard transformation, it follows that for all $i \in G, Y_{i} \supseteq \mathcal{R}_{i}(w)$. Hence, $X=\bigcap_{i \in G} Y_{i} \supseteq$ $\bigcap \mathcal{R}_{i}(w)=\mathcal{R}_{G}(w)$.

() Suppose that $\mathcal{R}_{G}(w) \subseteq X$. So $\bigcap_{i \in G} \mathcal{R}_{i}(w) \subseteq X$. For all $i \in G$, let $Y_{i}=$ $\mathcal{R}_{i}(w) \cup X$. Note that $\bigcap_{i \in G} Y_{i}=\left(\bigcap_{i \in G} \mathcal{R}_{i}(w)\right) \cup X=X$. Since each $\mathcal{N}_{\mathcal{R}_{i}}$ is obtained from $\mathcal{R}_{i}$ by the standard transformation, it follows that each $Y_{i} \in \mathcal{N}_{\mathcal{R}_{i}}(w)$. Hence, $X=\bigcap_{i \in G} Y_{i} \in \cap_{i \in G} \mathcal{N}_{\mathcal{R}_{i}}(w)$.

Theorem 1 Let $\mathfrak{S}$ be a relational model with domain $W$. Then $\mathfrak{S}$ and $\mathfrak{M}_{\mathfrak{S}}$ are $\mathfrak{L}_{G}^{[\forall]}$-equivalent: for all $w \in W$ and all $\varphi \in \mathfrak{L}_{G}^{[\forall]}, \mathfrak{S}, w \models \varphi$ iff $\mathfrak{M}_{\mathfrak{S}}, w \models \varphi$.

Proof By an induction on the complexity of $\varphi$. We only consider the case where $\varphi=$ $\square_{G} \psi$. Here we have: $\mathfrak{S}, w \models \varphi$ iff [by Definition 9] $\mathcal{R}_{G}(w) \subseteq\|\psi\|^{\mathfrak{S}}$ iff [by Lemma

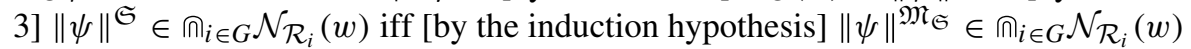
iff [by Definition 2] $\mathfrak{M}_{\mathfrak{S}}, w \models \varphi$.

Lemma 4 Let $\mathfrak{M}=\left\langle W,\left\langle\mathcal{N}_{i}\right\rangle_{i \in I}, V\right\rangle$ be an augmented neighbourhood model. Then, for all $w \in W$,

$$
\mathcal{N}_{G}(w)=\left\{X \subseteq W \mid \bigcap_{i \in G} \mathcal{R}_{\mathcal{N}_{i}}(w) \subseteq X\right\}
$$

Proof ( $\subseteq$ ) Suppose $X \in \mathcal{N}_{G}(w)$. Hence there is a set $\left\{Y_{i} \mid i \in G, Y_{i} \in \mathcal{N}_{i}(w)\right\}$ such that $\bigcap Y_{i}=X$. Note that by the standard transformation, for every $i \in G$, $\mathcal{R}_{\mathcal{N}_{i}}(w) \subseteq Y_{i}$. Consequently, $\bigcap_{i \in G} \mathcal{R}_{\mathcal{N}_{i}}(w) \subseteq X$.

() Suppose that $\bigcap_{i \in G} \mathcal{R}_{\mathcal{N}_{i}}(w) \subseteq X$. For every $i \in G$, let $Y_{i}=\mathcal{R}_{\mathcal{N}_{i}}(w) \cup X$. Note that, since each $\mathcal{N}_{i}$ is augmented, each $Y_{i} \in \mathcal{N}_{i}(w)$. Moreover, by the supposition, $X=\bigcap_{i \in G} Y_{i}$. Hence, $X \in \cap_{i \in G} \mathcal{N}_{i}(w)=\mathcal{N}_{G}(w)$.

Theorem 2 Let $\mathfrak{M}$ be an augmented neighbourhood model with domain $W$. Then $\mathfrak{S}_{\mathfrak{M}}$ is a relational model. Moreover, $\mathfrak{M}$ and $\mathfrak{S}_{\mathfrak{M}}$ are $\mathfrak{L}_{G}^{[\forall]}$-equivalent: for all $w \in W$ and all $\varphi \in \mathfrak{L}_{G}^{[\forall]}, \mathfrak{M}, w \models \varphi$ iff $\mathfrak{S}_{\mathfrak{M}}, w \models \varphi$.

Proof Analogous to the proof of Theorem 1, but relying on Lemma 4.

Taken together, Theorems 1 and 2 imply:

Corollary 1 For all $\varphi \in \mathfrak{L}_{G}^{[\forall]}, \varphi$ is valid on the class of all relational models iff $\varphi$ is valid on the class of all augmented neighbourhood models.

\subsection{Normal Modal Logics as Fragments of Pooling Logics}

In view of the preceding, one can characterize normal modal logics with intersection modalities by means of neighbourhood semantics with pooling modalities, by 
restricting the class of models. In this section, we show that the same can already be done within the broader class of monotonic models, by using the full expressive power of the language $\mathfrak{L}_{\infty}^{[\forall]}$. 18

This requires some preparation. For every $G \in \mathbb{G}$, let $G^{\infty}=\{(i, \infty) \mid i \in$ $G\} \cup\{(i, 0) \mid i \notin G\}$. Note that $G^{\infty} \in \mathbb{M}_{\infty}$. We define the translation function $\operatorname{tr}: \mathfrak{L}_{G}^{[\forall]} \rightarrow \mathfrak{L}_{\infty}^{[\forall]}$ as follows:

$$
\begin{aligned}
& \operatorname{tr}(\varphi)=\varphi \text { for all } \varphi \in \mathfrak{P} \\
& \operatorname{tr}(\neg \varphi)=\neg \operatorname{tr}(\varphi) \\
& \operatorname{tr}(\varphi \vee \psi)=\operatorname{tr}(\varphi) \vee \operatorname{tr}(\psi) \\
& \operatorname{tr}([\forall] \varphi)=[\forall] \operatorname{tr}(\varphi) \\
& \operatorname{tr}\left(\square_{G} \varphi\right)=\bigvee_{\emptyset \subset H \subseteq G} \square_{H} \infty \operatorname{tr}(\varphi) \vee[\forall] \operatorname{tr}(\varphi)
\end{aligned}
$$

With every monotonic (multi-neighbourhood) model, we can associate a (multiagent) relational model, by putting $\mathcal{R}_{i}(w)=\bigcap\left(\mathcal{N}_{i}(w) \cup\{W\}\right)$ for all worlds $w$. With infinitary pooling modalities at our disposal, we can express all properties of that relational model that could be expressed by the corresponding normal modal operators, as we now show.

Definition 10 Let $\mathfrak{M}=\left\langle W,\left\langle\mathcal{N}_{i}\right\rangle_{i \in I}, V\right\rangle$ be a monotonic model. The unit-augmentation of $\mathfrak{M}$ is the model $\mathfrak{M}^{\bigcap}=\left\langle W,\left\langle\mathcal{N}_{i}^{\cap}\right\rangle_{i \in I}, V\right\rangle$, where for every $i \in I$ and every $w \in W, \mathcal{N}_{i}^{\cap}(w)$ is the closure of $\mathcal{N}_{i}(w) \cup\{W\}$ under arbitrary intersections, i.e., $\mathcal{N}_{i}^{\cap}(w)=\cap^{\infty}\left(\mathcal{N}_{i}(w) \cup\{W\}\right)$.

Note that the unit-augmentation of a given monotonic model $\mathfrak{M}$ is an augmented model. The following is an immediate consequence of the definition of the neighbourhoods $\mathcal{N}_{i}^{\cap: 19}$

Fact 5 Let $\mathfrak{M}=\left\langle W,\left\langle\mathcal{N}_{i}\right\rangle_{i \in I}, V\right\rangle$ be a monotonic model and let $\mathfrak{M}^{\bigcap}=\left\langle W,\left\langle\mathcal{N}_{i}^{\cap}\right\rangle_{i \in I}, V\right\rangle$ be its unit-augmentation. Let $G \subseteq I$ be non-empty and finite and let $w \in W$. If for all $i \in G, \mathcal{N}_{i}(w) \neq \emptyset$, then $\cap_{i \in G} \mathcal{N}_{i}^{\cap}(w)=\mathcal{N}_{G^{\infty}}(w)$.

Theorem 3 Let $\mathfrak{M}$ be a monotonic model. For all $w$ in the domain of $\mathfrak{M}$ and for all $\varphi \in \mathfrak{L}_{G}^{[\forall]}: \mathfrak{M}^{\bigcap}, w \models \varphi$ iff $\mathfrak{M}, w \models \operatorname{tr}(\varphi)$.

Proof By an induction on the complexity of $\varphi$. The inductive base $(\varphi \in \mathfrak{P})$ and the induction step for the connectives and $[\forall]$ are safely left to the reader. So let $\varphi=\square_{G} \psi$ and let $w \in W$, where $W$ is the domain of $\mathfrak{M}$. Let

\footnotetext{
${ }^{18}$ Without $[\forall]$, one can obtain similar results for the intermediate class of models that are monotonic and where, for every $w \in W$ and all $i \in I, W \in \mathcal{N}_{i}(w)$. Here the translation consists merely in replacing each $G$ with $G^{\infty}$, and hence $[\forall]$ is not required. The lemmas and theorems of this section can be rephrased in terms of such unit-monotonic models, for this simpler translation.

${ }^{19}$ Note the similarity between Fact 2 and Fact 5: both basically state that if neighbourhoods are already closed under arbitrary intersections, there is no difference between multisets and the sets of indexes from which they are built up.
} 
$H=\left\{i \in G \mid \mathcal{N}_{i}(w) \neq \emptyset\right\}$. Note that for all $\tau$, whenever $\mathfrak{M}, w \models[\forall] \tau$, then $\mathfrak{M}, w \models \square_{H} \tau$ since $\mathfrak{M}$ is monotonic ( $\star$ ).

Case 1: $H \neq \emptyset$. Note that $\cap_{i \in H} \mathcal{N}_{i}^{\cap}(w)=\cap_{i \in G} \mathcal{N}_{i}^{\cap}(w)$ (†). Hence, $\mathfrak{M}^{\bigcap}, w \models \varphi$ iff [by the semantic clause] $\|\psi\|^{\mathfrak{M}^{\bigcap}} \in \cap_{i \in G} \mathcal{N}_{i}^{\cap}(w)$ iff [by (†)] $\|\psi\|^{\mathfrak{M} \cap} \in \cap_{i \in H} \mathcal{N}_{i}^{\cap}(w)$ iff [by the induction hypothesis] $\|\operatorname{tr}(\psi)\|^{\mathfrak{M}} \in \cap_{i \in H} \mathcal{N}_{i}^{\bigcap}(w)$ iff [by Fact 5] $\|\operatorname{tr}(\psi)\|^{\mathfrak{M}} \in \mathcal{N}_{H^{\infty}}(w)$ iff [by the semantic clause and our choice of $H$, and by $(\star)] \mathfrak{M}, w \models \bigvee_{\emptyset \subset H^{\prime} \subseteq G} \square_{H^{\prime}} \operatorname{tr}(\psi) \vee[\forall] \operatorname{tr}(\psi)$ iff $\mathfrak{M}, w \models \operatorname{tr}(\varphi)$.

Case 2: $H=\emptyset$. Then $\mathcal{N}_{G}^{\cap}(w)=\{W\}$ and, for all $G^{\prime} \subseteq G$ with $G^{\prime} \neq \varnothing$ and all $\tau, \mathfrak{M}, w \forall \forall \square_{G^{\prime}} \tau(\ddagger)$. Hence, $\mathfrak{M}^{\bigcap}, w \models \square_{G} \psi$ iff $\|\psi\|^{\mathfrak{M}^{\cap}}=W$ iff [by the induction hypothesis] $\|\operatorname{tr}(\psi)\|^{\mathfrak{M}}=W$ iff $\mathfrak{M}, w \models[\forall] \operatorname{tr}(\psi)$ iff [by (‡)] $\mathfrak{M}, w \models \bigvee_{\emptyset \subset G^{\prime} \subseteq G} \square_{G^{\prime}} \operatorname{tr}(\psi) \vee[\forall] \operatorname{tr}(\psi)$ iff $\mathfrak{M}, w \models \operatorname{tr}(\varphi)$.

Lemma 6 If $\mathfrak{M}$ is augmented, then for all $\varphi \in \mathfrak{L}_{G}^{[\forall]}: \mathfrak{M} \models \varphi$ iff $\mathfrak{M} \models \operatorname{tr}(\varphi)$.

Proof By an induction on the complexity of $\varphi$. Again we should only worry about the case $\varphi=\square_{G} \psi$. Here we can rely on the fact that, if $\mathfrak{M}$ is augmented, then it validates (i) $\square_{G} \psi \leftrightarrow \square_{G^{\infty}} \psi$ due to intersection closure and (ii) $\square_{G^{\infty}} \psi \leftrightarrow\left(\square_{G^{\infty}} \psi \vee[\forall] \psi\right)$ due to upward closure.

Theorem 4 For all $\varphi \in \mathfrak{L}_{G}^{[\forall]}, \varphi$ is valid on the class of all relational models iff $\operatorname{tr}(\varphi)$ is valid on the class of all monotonic models.

Proof $(\Rightarrow)$ Suppose there is a monotonic $\mathfrak{M}$ and a $w$ in its domain such that $\mathfrak{M}, w \not \forall \operatorname{tr}(\varphi)$. By Theorem 3, $\mathfrak{M}^{\cap}, w \not \forall \varphi$. Note that $\mathfrak{M}^{\bigcap}$ is augmented. By Theorem 2, $\mathfrak{S}_{\mathfrak{M}}, w \not \models \varphi$. Hence, $\varphi$ is not valid on the class of all relational models.

$(\Leftarrow)$ Suppose there is a relational model $\mathfrak{S}$ and a world $w$ in its domain such that $\mathfrak{S}, w \not \models \varphi$. Note that $\mathfrak{M}_{\mathfrak{S}}$ is augmented and hence also monotonic. By Theorem 1, $\mathfrak{M}_{\mathfrak{S}}, w \not \models \varphi$. By Lemma $6, \mathfrak{M}_{\mathfrak{S}}, w \not \models \operatorname{tr}(\varphi)$. Hence $\operatorname{tr}(\varphi)$ is not valid on the class of all monotonic models.

\subsection{The Monotonic Semantic Clause}

As noted in the introduction, in this paper we focus on the exact semantic clause for the operators $\square_{M}: \square_{M} \varphi$ holds at $w$ iff the exact truth set of $\varphi$ is a member of the neighbourhood $\mathcal{N}_{M}(w)$. There is, however, a well-known connection between monotonic neighbourhood models and a different semantic clause, viz. the monotonic semantic clause. In this section we show how this connection generalizes to pointwise intersection and pooling modalities.

For the sake of convenience, let us introduce a new symbol $\models_{m}$ for truth at a world in a model according to the monotonic clause. This notion is defined in the same way as $\models$, except that we replace the semantic clause for $\square$ with the following:

Definition 11 Let $\mathfrak{M}=\left\langle W,\left\langle\mathcal{N}_{i}\right\rangle_{i \in I}, V\right\rangle$ be a model, $w \in W$, and $\varphi \in \mathfrak{L}_{\infty}^{[\forall]}$. Then $\mathfrak{M}, w=_{m} \square_{M} \varphi$ iff there is an $X \in \mathcal{N}_{M}(w)$ such that $X \subseteq\|\varphi\|^{\mathfrak{M}}$. 
It has been argued that a focus on monotonic neighbourhood models - or equivalently, using the monotonic semantic clause - allows for a smoother model theory [31].

In contrast, at the level of the semantic consequence relation, the exact reading offers a perspective that is strictly more general, since one can characterize that semantic consequence relation simply by imposing the frame condition of monotony. We will now show how this generalizes to languages that feature pooling modalities.

In the remainder, we use $\mathcal{N}_{M}^{\uparrow}$ to refer to the neighbourhood function for the pooling profile $M$, in the supplemented model $\mathfrak{M}^{\uparrow}$ (cf. Definition 4). That is, $\mathcal{N}_{M}^{\uparrow}=\left\{\bigcap_{i \in I(M)} X_{i} \mid X_{i} \in \cap^{M(i)} \mathcal{N}_{i}^{\uparrow}(w)\right\}$. The following lemma states that, essentially, it makes no difference whether we (a) first close all neighbourhood sets under supersets, and afterwards apply pointwise intersection, or (b) first apply pointwise intersection to a given tuple of neighbourhood sets, and only afterwards close the resulting neighbourhood set under supersets.

Lemma 7 Let $M \in \mathbb{M}_{\infty}$ and let $\mathfrak{M}=\left\langle W,\left\langle\mathcal{N}_{i}\right\rangle_{i \in I}, V\right\rangle$ be given. Then $\mathcal{N}_{M}^{\uparrow}(w)=$ $\left(\mathcal{N}_{M}(w)\right)^{\uparrow}$.

Proof ( $\subseteq$ ) Suppose that $Y \in\left\{\bigcap_{i \in I(M)} X_{i} \mid X_{i} \in \cap^{M(i)} \mathcal{N}_{i}^{\uparrow}(w)\right\}$. So for every $i \in I(M)$, for every $k \in\{1, \ldots, M(i)\}$, there is an $X_{i}^{k} \in \mathcal{N}_{i}^{\uparrow}(w)$ such that $\bigcap_{k \in\{1, \ldots, M(i)\}} X_{i}^{k}=X_{i}$, and $\bigcap_{i \in I(M)} X_{i}=Y$. For every such $k, i$, let $Z_{i}^{k} \in \mathcal{N}_{i}(w)$ be such that $Z_{i}^{k} \subseteq X_{i}^{k}$. It follows that $Z_{i}=\bigcap_{k \in\{1, \ldots, M(i)\}} Z_{i}^{k} \in \cap^{M(i)} \mathcal{N}_{i}(w)$ and hence $Z=\bigcap_{i \in I(M)} Z_{i} \in\left\{\bigcap_{i \in I(M)} X_{i} \mid X_{i} \in \cap^{M(i)} \mathcal{N}_{i}(w)\right\}$. Moreover, $Z \subseteq Y$ and hence $Y \in\left\{\bigcap_{i \in I(M)} X_{i} \mid X_{i} \in \cap^{M(i)} \mathcal{N}_{i}(w)\right\}^{\uparrow}$.

() Suppose that $Y \in\left\{\bigcap_{i \in I(M)} X_{i} \mid X_{i} \in \cap^{M(i)} \mathcal{N}_{i}(w)\right\}^{\uparrow}$. So there is an $X \in$ $\left\{\bigcap_{i \in I(M)} X_{i} \mid X_{i} \in \cap^{M(i)} \mathcal{N}_{i}(w)\right\}$ such that $X \subseteq Y$. So for all $i \in I(M)$, for all $k \in$ $\{1, \ldots, M(i)\}$, there is an $X_{i}^{k} \in \mathcal{N}_{i}(w)$ such that each $X_{i}=\bigcap_{k \in\{1, \ldots, M(i)\}} X_{i}^{k}$ and $\bigcap_{i \in I(M)} X_{i} \subseteq Y$. For all $i \in I(M)$ and all $k \in\{1, \ldots, M(i)\}$, let $Y_{i}^{k}=Y \cup X_{i}^{k}$. Then in view of the preceding, each $Y_{i}^{k} \in \mathcal{N}_{i}^{\uparrow}(w)$. Also, $Y=\bigcap_{i \in I(M), k \in\{1, \ldots, M(i)\}} Y_{i}^{k}$. Hence, $Y \in\left\{\bigcap_{i \in I(M)} X_{i} \mid X_{i} \in \cap^{M(i)} \mathcal{N}_{i}^{\uparrow}(w)\right\}$.

By a standard induction on the complexity of formulas, we obtain:

Corollary 2 Where $\mathfrak{M}=\left\langle W,\left\langle\mathcal{N}_{i}\right\rangle_{i \in I}, V\right\rangle$ is a model, $w \in W$, and $\varphi \in \mathfrak{L}_{\infty}^{[\forall]}$ : $\mathfrak{M}, w \models_{m} \varphi$ iff $\mathfrak{M}^{\uparrow}, w \models \varphi$.

Hence, the logic obtained by using the monotonic semantic clause for $\square_{M}$ is just the same as the logic of the restricted class of models in which the neighbourhood sets are closed under supersets. In [43], this insight is put to work in the completeness proof for monotonic modal logics with pooling modalities.

Of course, this does not take away that there are other, independent reasons for working with the monotonic clause: e.g. when the neighbourhoods are also used to define other operators in a given formal language. A prominent example is Evidence 
Logic [3, 40], in which the monotonic clause is used to interpret an operator expressing evidence, but the neighbourhoods are also used to interpret evidence-based belief (in terms of maximal non-empty intersections of exact pieces of evidence). Having the neighbourhood sets closed under supersets in this setting would ruin the logic of belief, in the sense that inconsistent evidence would always result in having only tautological beliefs.

It should be noted here that there is a yet different characterization of monotonic modal logics, viz. in terms of multirelational semantics $[15,16]$. Over multirelational models, intersection of the accessibility relations corresponds to pointwise intersection of neighbourhood sets. ${ }^{20}$ In sum, monotonic models (using the exact semantic clause), arbitrary models (using the monotonic semantic clause), and multirelational models (using the multi-relational semantic clause) can all be used to interpret the same logic of pooling modalities.

\section{Expressivity}

In this section we map out the expressive power of the various formal languages introduced so far. We first show that, for the six formal languages considered in this paper, proper inclusion of the languages also entails a proper difference in expressive power at the level of models. We then consider one specific condition on models under which all pooled information is expressible in the languages without pooling modalities, linking this to existing work in epistemic logic on the so-called principle of "full communication". Finally, we move to the level of frames, showing that also there, pooling modalities yield a surplus in expressive power.

\subsection{Expressivity at the Level of Models}

Let a pointed model be a pair $(\mathfrak{M}, w)$, where $w$ is a member of the domain of $\mathfrak{M}$. Following a customary practice in modal logic, one may identify the expressive power of a language with the distinctions it can make among pointed models. In order to apply this criterion, we specify first what it means that two pointed models are indistinguishable from the viewpoint of a given formal language $\mathfrak{T}$.

Definition 12 Let $\mathfrak{T} \in\left\{\mathfrak{L}, \mathfrak{L}^{[\forall]}, \mathfrak{L}_{f}, \mathfrak{L}_{f}^{[\forall]}, \mathfrak{L}_{\infty}, \mathfrak{L}_{\infty}^{[\forall]}\right\}$. Let $\mathfrak{M}=\left\langle W,\left\langle\mathcal{N}_{i}\right\rangle_{i \in I}, V\right\rangle$ and $\mathfrak{M}^{\prime}=\left\langle W^{\prime},\left\langle\mathcal{N}_{i}^{\prime}\right\rangle_{i \in I}, V^{\prime}\right\rangle$ be models and let $w \in W, w^{\prime} \in W^{\prime}$. Then $(\mathfrak{M}, w) \equiv \mathfrak{T}$ $\left(\mathfrak{M}^{\prime}, w^{\prime}\right)$ iff for all $\varphi \in \mathfrak{T}, \mathfrak{M}, w \models \varphi$ iff $\mathfrak{M}^{\prime}, w^{\prime} \models \varphi$.

Where $\mathfrak{T}, \mathfrak{T}^{\prime}$ are formal languages, we write $\mathfrak{T} \leq \mathfrak{T}^{\prime}$ to indicate that $\mathfrak{T}^{\prime}$ is at least as expressive as $\mathfrak{T}$. That is: $\mathfrak{T} \leq \mathfrak{T}^{\prime}$ iff, for all $(\mathfrak{M}, w)$ and $\left(\mathfrak{M}^{\prime}, w^{\prime}\right)$ : if $(\mathfrak{M}, w) \equiv \mathfrak{T}^{\prime}$ $\left(\mathfrak{M}^{\prime}, w^{\prime}\right)$, then $(\mathfrak{M}, w) \equiv \mathfrak{T}\left(\mathfrak{M}^{\prime}, w^{\prime}\right)$. If the converse fails, we say that $\mathfrak{T}^{\prime}$ is strictly more expressive than $\mathfrak{T}$, formally: $\mathfrak{T}<\mathfrak{T}^{\prime}$.

\footnotetext{
${ }^{20}$ Spelling out this claim in full generality and proving it is somewhat tedious but can be safely left to the reader.
} 
The expressivity relations between the six languages considered in this paper are summarized by the following theorem, illustrated in Fig. 2

Theorem 5 For all $\mathfrak{T}, \mathfrak{T}^{\prime} \in\left\{\mathfrak{L}, \mathfrak{L}^{[\forall]}, \mathfrak{L}_{f}, \mathfrak{L}_{f}^{[\forall]}, \mathfrak{L}_{\infty}, \mathfrak{L}_{\infty}^{[\forall]}\right\}: \mathfrak{T} \leq \mathfrak{T}^{\prime}$ iff $\mathfrak{T} \subseteq \mathfrak{T}^{\prime}$.

Obviously, if $\mathfrak{T} \subseteq \mathfrak{T}^{\prime}$, then $\mathfrak{T} \leq \mathfrak{T}^{\prime}$. It order to show the converse, it suffices to observe three basic facts.

Fact $8 \mathfrak{L}^{[\forall]} \not \leq \mathfrak{L}_{\infty}$.

Let us illustrate this fact by means of a simple example for $I=\{1\}$. Let $\mathfrak{M}=\left\langle W,\left\langle\mathcal{N}_{i}\right\rangle_{i \in I}, V\right\rangle$ with $W=\{w, v\}, \mathcal{N}_{1}(w)=\mathcal{N}_{1}(v)=\{\{w\}\}, V(p)=\{w\}$, and $V(q)=\emptyset$. Let $\mathfrak{M}^{\prime}=\left\langle W^{\prime},\left\langle\mathcal{N}_{i}^{\prime}\right\rangle_{i \in I}, V^{\prime}\right\rangle$ with $W^{\prime}=\left\{w^{\prime}, v^{\prime}, u^{\prime}\right\}, \mathcal{N}_{1}^{\prime}\left(w^{\prime}\right)=$ $\mathcal{N}_{1}^{\prime}\left(v^{\prime}\right)=\mathcal{N}_{1}^{\prime}\left(u^{\prime}\right)=\left\{w^{\prime}\right\}, V^{\prime}(p)=\left\{w^{\prime}\right\}$, and $V^{\prime}(q)=\left\{u^{\prime}\right\}$. These two models are depicted in Fig. 3. It can easily be verified by an induction that $(\mathfrak{M}, w) \equiv \mathfrak{L}_{\infty}$ $\left(\mathfrak{M}^{\prime}, w^{\prime}\right)$. However, $\mathfrak{M}, w \models[\forall] \neg q$, whereas $\mathfrak{M}^{\prime}, w^{\prime}=[\exists] q$.

In the context of non-normal modal logics - which allow us to model i.a. agents having inconsistent information or normative systems that issue incompatible norms - , the universal modality is particularly useful, in that it allows us to express that two or more propositions are jointly compatible. For instance, using a non-normal modality $\square_{1}$ we may express that agent 1 has evidence for $p$ by $\square_{1} p$, and that this same agent has evidence for $q\left(\square_{1} q\right)$. The existential modality then allows us to express that moreover, these pieces of evidence are jointly compatible: $[\exists](p \wedge q)$.

Fact $9 \mathfrak{L}_{f} \not \mathfrak{L}^{[\forall]}$.

As argued in Section 4, pointwise intersection provides a conservative generalization of the intersection of accessibility relations in relational semantics. It is a well-known fact that, for relational semantics, adding intersection modalities makes

Fig. 2 Hasse diagram representing the expressive power of the six languages, cf. Theorem 5

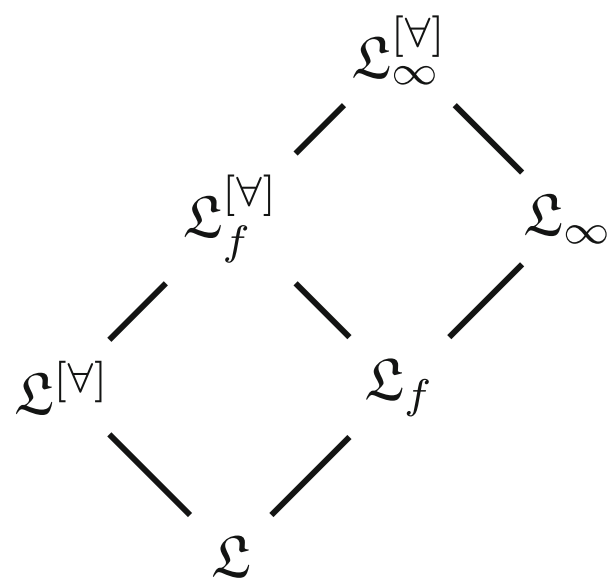


$\mathfrak{M}$

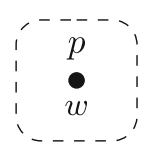

v
$\mathfrak{M}^{\prime}$

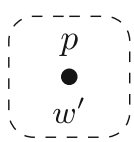

Fig. 3 Two pointed models $(\mathfrak{M}, w)$ and $\left(\mathfrak{M}^{\prime}, w^{\prime}\right)$ that are $\mathfrak{L}_{\infty}$-equivalent but not $\mathfrak{L}^{[\forall]}$-equivalent. The dashed set denotes the agent's (only) neighborhood at all worlds

for a strict increase in expressive power. As a corollary, within the class of augmented models, one can already find models that are distinguishable only by means of (finitary) pooling modalities. ${ }^{21}$

In the general class of neighbourhood models, we can also illustrate the expressive power of finitary pooling modalities by two single-agent models. That is, let $\mathfrak{M}=\left\langle W, \mathcal{N}_{1}, V\right\rangle$ with $W=\{w, v\}, \mathcal{N}_{1}(w)=\mathcal{N}_{1}(v)=\{\{w\},\{v\}\}$, and $V(p)=\varnothing$ for all $p \in \mathfrak{P}$. Let $\mathfrak{M}^{\prime}=\left\langle W^{\prime}, \mathcal{N}_{1}^{\prime}, V^{\prime}\right\rangle$ with $W^{\prime}=\left\{w^{\prime}, v^{\prime}, u^{\prime}\right\}, \mathcal{N}_{1}^{\prime}\left(w^{\prime}\right)=\mathcal{N}_{1}^{\prime}\left(v^{\prime}\right)=$ $\mathcal{N}_{1}^{\prime}\left(u^{\prime}\right)=\left\{\left\{w^{\prime}, v^{\prime}\right\},\left\{v^{\prime}, u^{\prime}\right\}\right\}$, and $V^{\prime}(p)=\emptyset$ for all $p \in \mathfrak{P}$. These two pointed models are depicted in Fig. 4. Note that none of the neighbourhoods in these models correspond to the truth set of some formula. Relying on this insight, it can easily be verified that $\mathfrak{M}, w \equiv_{\mathfrak{L}[\forall]} \mathfrak{M}^{\prime}, w^{\prime}$. In the first model, $\square_{1,1} \perp$ holds, whereas in the second it fails. ${ }^{22}$

Fact $10 \mathfrak{L}_{\infty} \not \mathfrak{L}_{f}^{[\forall]}$.

The step from finitary pooling modalities to arbitrary pooling modalities is easily made, conceptually speaking. However, it again results in a strict increase in expressive power. To see why, consider the single-index example given by Fig. 5. Here, for every $w_{i} \in W, \mathcal{N}_{1}\left(w_{i}\right)$ consists of only one set, i.e. the unit $W$. In contrast, for every $w_{j}^{\prime} \in W^{\prime}, \mathcal{N}_{1}^{\prime}\left(w_{j}^{\prime}\right)$ contains $W^{\prime}$ and, in addition, an infinite series of ever smaller sets $X_{n}=W^{\prime} \backslash\left\{w_{1}^{\prime}, \ldots, w_{n}^{\prime}\right\}$. Since there is no proposition that distinguishes any of the worlds $w_{i}^{\prime}$, the $X_{i}$ do not correspond to the truth set of any formula in $\mathfrak{L}_{f}^{[\forall]}$. The same holds for all sets $\bigcap_{i \leq k} X_{i}$ for $k \in \mathbb{N}^{+}$. Relying on this observation one can easily show that $\left(\mathfrak{M}, w_{i}\right) \equiv_{\mathfrak{L}_{f}^{[\forall] ~}}\left(\mathfrak{M}^{\prime}, w_{j}^{\prime}\right)$ for all $i, j \in \mathbb{N}$. However, for every $i \in \mathbb{N}$, $\mathfrak{M}, w_{i} \not \models \square_{1} \perp$ but, since $\bigcap_{i \in \mathbb{N}} X_{i}=\emptyset, \mathfrak{M}^{\prime}, w_{j}^{\prime} \models \square_{1} \perp$ for every $j \in \mathbb{N}$.

It is no coincidence that the model $\mathfrak{M}^{\prime}$ in Fig. 5 has an infinite domain. That is, suppose $\mathfrak{M}$ has finite domain $W$. Then there can be at most $k=|\wp(W)|$ many different subsets of $W$. Hence any intersection of a set of subsets of $W$ can be rewritten

\footnotetext{
${ }^{21}$ In fact, the mentioned pooling profiles $M$ are even more specific, since they correspond to regular sets, i.e., $M(i)=1$ for all $i \in I(M)$.

${ }^{22}$ The same observations can be made about the supplementation of $\mathfrak{M}$ and $\mathfrak{M}$, obtained by closing their respective neighbourhood sets under supersets.
} 
$\mathfrak{M}$

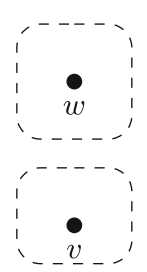

$\mathfrak{M}^{\prime}$

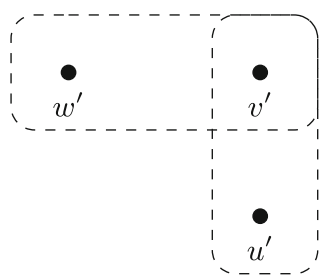

Fig. 4 Two pointed models $(\mathfrak{M}, w)$ and $\left(\mathfrak{M}^{\prime}, w^{\prime}\right)$ that are $\mathfrak{L}^{[\forall]}$-equivalent but not $\mathfrak{L}_{f}$-equivalent

as an intersection of $k$ subsets of $W$. So, letting $M_{k}=\{(i, k) \mid M(i)=\infty\} \cup\{(j, l) \mid$ $M(j)=l \neq \infty\}$, it follows that $\mathfrak{M}, w \models \square_{M} \varphi$ iff $\mathfrak{M}, w \models \square_{M_{k}} \varphi$ for all $\varphi \in \mathfrak{L}_{\infty}^{[\forall]}$. So whatever can be expressed about finite models using formulas in $\mathfrak{L}_{\infty}\left(\mathfrak{L}_{\infty}^{[\forall]}\right)$ can also be expressed using formulas in $\mathfrak{L}_{f}\left(\mathfrak{L}_{f}^{[\forall]}\right)$. Consequently:

Fact 11 Suppose that $\mathfrak{M}$ and $\mathfrak{M}^{\prime}$ are finite. Then each of the following hold:

1. $(\mathfrak{M}, w) \equiv \mathfrak{L}_{\infty}\left(\mathfrak{M}^{\prime}, w^{\prime}\right)$ iff $(\mathfrak{M}, w) \equiv \mathfrak{L}_{f}\left(\mathfrak{M}^{\prime}, w^{\prime}\right)$

2. $(\mathfrak{M}, w) \equiv_{\mathfrak{L}_{\infty}^{[\forall]}}\left(\mathfrak{M}^{\prime}, w^{\prime}\right)$ iff $(\mathfrak{M}, w) \equiv_{\mathfrak{L}_{f}^{[\forall]}}\left(\mathfrak{M}^{\prime}, w^{\prime}\right)$.

\subsection{Differentiability and Full Communication}

In the preceding we saw that pooling modalities add expressive power to $\mathfrak{L}$ and $\mathfrak{L}^{[\forall]}$. In metaphoric terms, the pooled information $\varphi$ - say, the beliefs of different agents - may sometimes stay "under the radar", since it is not witnessed by any individual pieces $\psi_{1}, \psi_{2}, \ldots$ of information that, when combined, yield $\varphi$. In the present section, we delineate a specific class of models in which such witnesses are always available, and show that for this class, $\mathfrak{L}$ and $\mathfrak{L}^{[\forall]}$ are as expressive as their extensions with pooling modalities. Next, we show that such models also satisfy a principle that is well-known from the study of normal doxastic and epistemic logics.

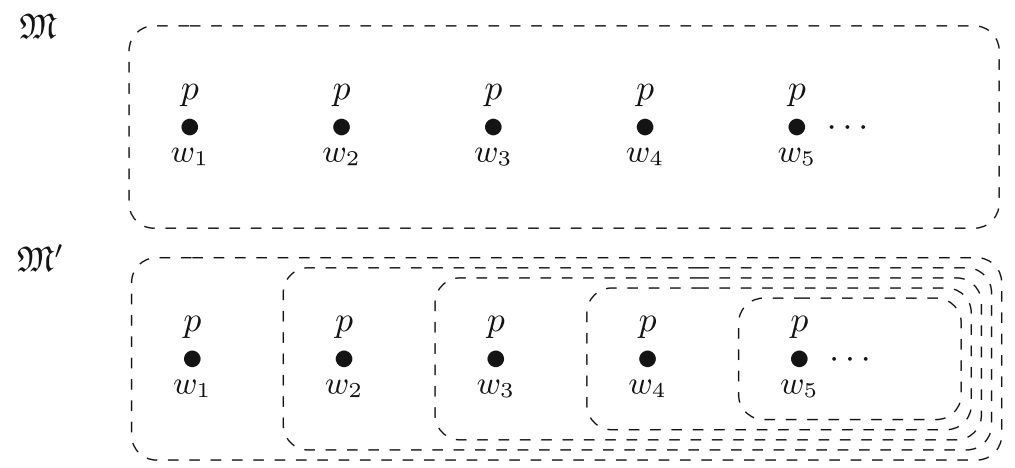

Fig. 5 Two pointed models $\left(\mathfrak{M}, w_{1}\right)$ and $\left(\mathfrak{M}^{\prime}, w_{1}^{\prime}\right)$ that are $\mathfrak{L}_{f}^{[\forall]}$-equivalent, but not $\mathfrak{L}_{\infty}$-equivalent 
Let us start by defining the notion of differentiability, cf. [2, 14, 34]. ${ }^{23}$

Definition $13 \mathfrak{M}=\left\langle W,\left\langle\mathcal{N}_{i}\right\rangle_{i \in I}, V\right\rangle$ is $\mathfrak{T}$-differentiable iff for every $w \in W$, for every $i \in I$, and for every $X \in \mathcal{N}_{i}(w)$, there is a $\varphi \in \mathfrak{T}$ such that $X=\|\varphi\|^{\mathfrak{M}}$.

Intuitively, that $\mathfrak{M}$ is $\mathfrak{T}$-differentiable means that every neighbourhood (of every world $w$, for every index $i$ ) in the model can be "named" by some formula in $\mathfrak{T}$.

Theorem 6 Suppose that $\mathfrak{M}$ and $\mathfrak{M}^{\prime}$ are both finite and $\mathfrak{L}^{[\forall]}$-differentiable. If $(\mathfrak{M}, w) \equiv_{\mathfrak{L}^{[\forall]}}\left(\mathfrak{M}^{\prime}, w^{\prime}\right)$, then $(\mathfrak{M}, w) \equiv_{\mathfrak{L}_{\infty}^{[\forall]}}\left(\mathfrak{M}^{\prime}, w^{\prime}\right)$

Proof Suppose that $\mathfrak{M}$ and $\mathfrak{M}^{\prime}$, with respective finite domains $W$ and $W^{\prime}$, are both $\mathfrak{L}^{[\forall]}$-differentiable and $(\mathfrak{M}, w) \equiv \sum_{\mathfrak{L}[\forall]}\left(\mathfrak{M}^{\prime}, w^{\prime}\right)$. We first prove the following:

$(\dagger 1)$ for all $v \in W$, there is a $v^{\prime} \in W^{\prime}$ such that $(\mathfrak{M}, v) \equiv_{\mathfrak{L}^{[\forall]}}\left(\mathfrak{M}^{\prime}, v^{\prime}\right)$

$\left(\dagger_{2}\right)$ for all $v^{\prime} \in W^{\prime}$, there is a $v \in W$ such that $(\mathfrak{M}, v) \equiv_{\mathfrak{L}^{[\forall]}}\left(\mathfrak{M}^{\prime}, v^{\prime}\right)$

To see why ( $\left.\dagger_{1}\right)$ holds, assume for contradiction that $v \in W$ but, for no $v^{\prime} \in W^{\prime}$, $v \equiv_{\mathfrak{L}^{[\forall]}} v^{\prime}$. So for all $v^{\prime} \in W^{\prime}$, some $\psi_{v^{\prime}} \in \mathfrak{L}^{[\forall]}$ is true at $v^{\prime}$ in $\mathfrak{M}^{\prime}$, but false $v$ in $\mathfrak{M}$. It follows that the conjunction $\bigwedge_{v^{\prime} \in W^{\prime}} \neg \psi_{v^{\prime}}$ is true at $v$ in $\mathfrak{M}$ and hence, $\mathfrak{M}, w \models[\exists] \bigwedge_{v^{\prime} \in W^{\prime}} \neg \psi_{v^{\prime}}$. However, in view of the preceding, $\mathfrak{M}^{\prime}, w^{\prime} \models$ $[\forall] \bigvee_{v^{\prime} \in W^{\prime}} \psi_{v^{\prime}}$. This contradicts the supposition that $(\mathfrak{M}, w) \equiv_{\mathfrak{L}[\forall]}\left(\mathfrak{M}^{\prime}, w^{\prime}\right)$. The proof for $\left(\dagger_{2}\right)$ is entirely analogous. Let us denote the conjunction of $\left(\dagger_{1}\right)$ and $\left(\dagger_{2}\right)$ by $(\dagger)$.

We prove by induction that, for all $v, v^{\prime} \in W \times W^{\prime}$ with $v \equiv_{\mathfrak{L}^{[\forall]}} v^{\prime}$, for all $\varphi \in \mathfrak{L}_{\infty}^{[\forall]}$, $\mathfrak{M}, v \models \varphi$ iff $\mathfrak{M}^{\prime}, v^{\prime} \models \varphi$. The base case $(\varphi \in \mathfrak{P})$ and the induction step for the connectives are trivial. For $\varphi=[\forall] \psi$, we have that $\mathfrak{M}, v \models \varphi$ iff for all $u \in W$, $\mathfrak{M}, u \models \psi$ iff [by the induction hypothesis and $(\dagger)$ ] for all $u^{\prime} \in W^{\prime}, \mathfrak{M}^{\prime}, u^{\prime} \models \psi$ iff $\mathfrak{M}^{\prime}, v^{\prime} \models \varphi$.

For $\varphi=\square_{M} \psi$, note first that by induction hypothesis (IH) and the induction step for the classical connectives and $[\forall]$ :

$$
\text { for all } \tau \in \mathfrak{L}^{[\forall]}, \mathfrak{M}, v \models[\forall](\tau \leftrightarrow \psi) \text { iff } \mathfrak{M}^{\prime}, v^{\prime} \models[\forall](\tau \leftrightarrow \psi)
$$

Assume now that $\mathfrak{M}, v \models \square_{M} \psi$ for some $v \in W$ and that $v \equiv_{\mathfrak{L}^{[\forall]}} v^{\prime}$. By the semantic clause for $\square_{M}$, for every $i \in I(M)$, there must be a (finite) set $\mathcal{X}_{i} \subseteq \mathcal{N}_{i}(v)$, such that $\bigcap_{i \in I(M)} \bigcap \mathcal{X}_{i}=\|\psi\|^{\mathfrak{M}}$. Since $\mathfrak{M}$ is $\mathfrak{L}^{[\forall]}$-differentiable, for every $i \in$ $I(M)$, for every $X \in \mathcal{X}_{i}$, there is a $\tau_{X} \in \mathfrak{L}^{[\forall]}$ such that $X=\left\|\tau_{X}\right\|^{\mathfrak{M}}$. Hence, for every such $X, \mathfrak{M}, v \models \square_{i} \tau_{X}$. Note that

$$
\bigcap_{i \in I(M)} \bigcap \mathcal{X}_{i}=\bigcap_{i \in I(M)} \bigcap_{X \in \mathcal{X}_{i}}\left\|\tau_{X}\right\|^{\mathfrak{M}}
$$

Let $\tau=\bigwedge_{i \in I(M)} \bigwedge_{X \in \mathcal{X}_{i}} \tau_{X}$. So we have:

$$
\|\tau\|^{\mathfrak{M}}=\|\psi\|^{\mathfrak{M}}
$$

\footnotetext{
${ }^{23}$ We borrow terminology from [2], but relativize our definition to the underlying language. In other papers, differentiable models are called "locally distinguishing" (with respect to a multi-agent epistemic language), cf. [14, 34].
} 
It follows that $\mathfrak{M}, v \models[\forall](\tau \leftrightarrow \psi)$ and hence also $\mathfrak{M}^{\prime}, v^{\prime} \models[\forall](\tau \leftrightarrow \psi)(\star)$.

By the supposition that $v \equiv_{\mathfrak{L}^{[\forall]}} v^{\prime}$, for every $\tau_{X}$ (with $X \in \mathcal{X}_{i}, i \in I(M)$ ), $\mathfrak{M}^{\prime}, v^{\prime} \models \square_{i} \tau_{X}$. Consequently, $\mathfrak{M}^{\prime}, v^{\prime}=\square_{M} \tau$. By $(\star), \mathfrak{M}^{\prime}, v^{\prime} \models \square_{M} \psi$. The proof for the other half of the equivalence is entirely analogous.

Let us briefly return to one of the potential applications of pooling modalities, i.e. the specification of non-normal logics for distributed information of agents and groups of agents. In this context, the principle of full communication [14, 34, 44] roughly says that $\varphi$ can be a piece of group knowledge only if there is some way the agents in $G$ would be able to arrive at the knowledge that $\varphi$ by communicating each of their individual pieces of knowledge. One plausible formal explication of this principle reads: ${ }^{24}$

(FC) for all $\varphi \in \mathfrak{L}^{[\forall]}$ : if $\mathfrak{M}, w \models \square_{G^{\infty}} \varphi$, then $\left\{\psi \in \mathfrak{L}^{[\forall]} \mid \mathfrak{M}, w \models\right.$ $\square_{i} \psi$ for some $\left.i \in G\right\} \Vdash \varphi$

A first thing to note is that (FC) fails on the class of all models. That is because (FC) fails on relational semantics for distributed knowledge, and more generally, on any relational semantics that interprets $\square_{G^{\infty}}$ by referring to the intersection of the relations $\mathcal{R}_{i}$ that correspond to $\square_{i}[14,34,44]$. The failure of (FC) for pooling modalities over (augmented) neighbourhood models follows from these known results as a corollary, in light of Section 4.1.

In [34], an overview of increasingly weak sufficient conditions for (FC) is given. Most of these conditions cannot easily be generalized to neighbourhood semantics, as they rely on the consistency of the agent-relative information sets $K S_{i}=\{\psi \in$ $\left.\mathfrak{L}^{[\forall]} \mid \mathfrak{M}, w \models \square_{i} \psi\right\}$. One exception is the condition of differentiability relative to $\mathfrak{L}^{[\forall]}$.

Theorem 7 If $\mathfrak{M}$ is finite and $\mathfrak{L}^{[\forall]}$-differentiable, then $(F C)$ holds for all $w$ in the domain of $\mathfrak{M}$.

Proof Suppose the antecedent holds and $\mathfrak{M}, w \models \square_{G^{\infty}} \varphi$ for some $\varphi \in \mathfrak{L}^{[\forall]}$. Since $W$ is finite, there are $X_{1}, \ldots, X_{n}$ and $i_{1}, \ldots, i_{n} \in G$ such that each $X_{j} \in \mathcal{N}_{i_{j}}(w)$ and $X_{1} \cap \ldots \cap X_{n}=\|\varphi\|^{\mathfrak{M}}$. Since $\mathfrak{M}$ is differentiable, for each $X_{j}$ there is a $\psi_{j} \in \mathfrak{L}^{[\forall]}$ such that $X_{j}=\left\|\psi_{j}\right\|^{\mathfrak{M}}$ and hence $\mathfrak{M}, w \models \square_{i_{j}} \psi_{j}$. It follows that $\left\|\bigwedge_{1 \leq j \leq n} \psi_{j}\right\|=\|\varphi\|$ and hence $\left\|\bigwedge_{1 \leq j \leq n} \psi_{j} \rightarrow \varphi\right\|^{\mathfrak{M}}=W$. Hence, for all $j \in\{1, \ldots, n\},\left\|\psi_{j}\right\|^{\mathfrak{M}}=\left\|\psi_{j} \wedge\left(\bigwedge_{1 \leq j \leq n} \psi_{j} \rightarrow \varphi\right)\right\|^{\mathfrak{M}}$. It follows that, for all $j \in\{1, \ldots, n\}$,

$$
\mathfrak{M}, w \models \square_{i_{j}}\left(\psi_{j} \wedge\left(\bigwedge_{1 \leq j \leq n} \psi_{j} \rightarrow \varphi\right)\right)
$$

\footnotetext{
${ }^{24}$ Over monotonic models, the convere of (FC) is always valid; for arbitrary models it may fail. Obvious ways to vary on (FC) concern the language in which the $\psi$ 's are contained. We choose $\mathfrak{L}^{[\forall]}$ here for the sake of generality and simplicity.
} 
For all $j \in\{1, \ldots, n\}$, let $\tau_{j}=\psi_{j} \wedge\left(\bigwedge_{1 \leq j \leq n} \psi_{j} \rightarrow \varphi\right)$. We have: $\left\{\tau_{j} \mid j \in\right.$ $\{1, \ldots, n\}\} \Vdash \varphi$ and for every $j \in\{1, \ldots, n\}, \mathfrak{M}, w \models \square_{i_{j}} \tau_{j}$. Hence (FC) holds for $w$ in $\mathfrak{M}$.

In conclusion, the class of finite, $\mathfrak{L}^{[\forall]}$-differentiable models constitutes a proper subclass of the class of all models, over which pooling modalities add no expressive power and full communication is valid.

\subsection{Expressivity at the Level of Frames}

Besides studying the expressive power of various formal languages at the level of models, one may also compare their expressive power in terms of frames. Let us first fix terminology. A pointed frame is a pair $(\mathfrak{F}, w)$, where $\mathfrak{F}$ is a frame and $w$ is a member of the domain of $\mathfrak{F}$. Where $W$ is the domain of $\mathfrak{F}, \mathfrak{F}, w \models \varphi$ iff for all valuations $V: W \rightarrow \wp(\mathfrak{P}),\langle\mathfrak{F}, V\rangle, w \models \varphi$.

We say that $\varphi \in \mathfrak{L}_{\infty}^{[\forall]}$ characterizes the frame condition (C) iff the following equivalence holds, for all pointed frames $(\mathfrak{F}, w)$ :

$$
\mathfrak{F}, w \models \varphi \text { iff }(\mathfrak{F}, w) \text { satisfies }(\mathrm{C})
$$

In other words, $(\mathfrak{F}, w)$ does not satisfy the given frame condition if and only if one can find a valuation $V$ over $\mathfrak{F}$ such that, given $V, \varphi$ is false at $w$. For the sake of convenience we will state our results in terms of pointed frames, since the conditions we consider typically refer to neighbourhood sets of a given world. One can however easily derive corollaries that apply to frames more generally. In particular, if $\varphi$ characterizes the frame condition $(\mathrm{C})$, then it follows that $\varphi$ is valid on a frame $\mathfrak{F}$ iff condition (C) holds at every world in $\mathfrak{F} .{ }^{25}$

We give two relatively simple examples to show that, also from the perspective of frames, pooling modalities give us more expressive power.

Theorem 8 Let $\mathfrak{F}=\left\langle W,\left\langle\mathcal{N}_{i}\right\rangle_{i \in I}\right\rangle$ be a frame and let $w \in W$. Then $\mathcal{N}_{2}(w) \subseteq$ $\mathcal{N}_{1}(w) \cap \mathcal{N}_{1}(w)$ iff $\mathfrak{F}, w=\square_{2} p \rightarrow \square_{1,1} p$. Moreover, this frame condition cannot be characterized within $\mathfrak{L}^{[\forall]}$.

Proof The proof of the first part of this theorem is safely left to the reader. For the second part, assume for contradiction that the frame condition can be characterized by a formula $\varphi \in \mathfrak{L}^{[\forall]}$. Let $I=\{1,2\}, \mathfrak{F}=\left\langle W,\left\langle\mathcal{N}_{i}\right\rangle_{i \in I}\right\rangle$ with $W=\{w, v\}, \mathcal{N}_{1}(w)=$ $\mathcal{N}_{1}(v)=\varnothing$, and $\mathcal{N}_{2}(w)=\mathcal{N}_{2}(v)=\{\{w\}\}$. Note that the pointed frame $(\mathfrak{F}, w)$ does not satisfy the mentioned frame condition. Let $V: W \rightarrow \wp(\mathfrak{P})$ be such that $\mathfrak{M}, w \not \models \neg \varphi$, where $\mathfrak{M}=\left\langle W,\left\langle\mathcal{N}_{i}\right\rangle_{i \in I}, V\right\rangle$.

Let now $\mathfrak{M}^{\prime}=\left\langle W^{\prime},\left\langle\mathcal{N}_{i}^{\prime}\right\rangle_{i \in I}, V^{\prime}\right\rangle$, where $W^{\prime}=\left\{w^{\prime}, v_{1}^{\prime}, v_{2}^{\prime}\right\}, \mathcal{N}_{1}^{\prime}\left(w^{\prime}\right)=\mathcal{N}_{1}^{\prime}\left(v_{1}^{\prime}\right)=$ $\mathcal{N}_{1}^{\prime}\left(v_{2}^{\prime}\right)=\left\{\left\{w^{\prime}, v_{1}^{\prime}\right\},\left\{w^{\prime}, v_{2}^{\prime}\right\}\right\}, \mathcal{N}_{2}^{\prime}\left(w^{\prime}\right)=\mathcal{N}_{2}^{\prime}\left(v_{1}^{\prime}\right)=\mathcal{N}_{2}^{\prime}\left(v_{2}^{\prime}\right)=\left\{\left\{w^{\prime}\right\}\right\}$, and $V^{\prime}\left(w^{\prime}\right)=V(w), V^{\prime}\left(v_{1}^{\prime}\right)=V^{\prime}\left(v_{2}^{\prime}\right)=V(v)$. Then one can show by an induction

\footnotetext{
${ }^{25}$ Following standard terminology, $\varphi$ is valid on $\mathfrak{F}$ iff for all $w$ in the domain of $\mathfrak{F}$ and all valuations $V$ over $\mathfrak{F},\langle\mathfrak{F}, V\rangle, w \models \varphi$.
} 
that $(\mathfrak{M}, w) \equiv_{\mathfrak{L}^{[\forall]}}\left(\mathfrak{M}^{\prime}, w^{\prime}\right)$ and hence $\mathfrak{M}^{\prime}, w^{\prime} \models \neg \varphi$. (For the induction step with $\varphi=\square_{1} \psi$, observe that neither $\left\{w^{\prime}, v_{1}^{\prime}\right\}$, nor $\left\{w^{\prime}, v_{2}^{\prime}\right\}$ are definable in the model $\mathfrak{M}^{\prime}$.) Note however that $\mathcal{N}_{2}^{\prime}\left(w^{\prime}\right)=\mathcal{N}_{1}^{\prime}\left(w^{\prime}\right) \cap \mathcal{N}_{1}^{\prime}\left(w^{\prime}\right)$, contradicting the fact that $\varphi$ expresses this frame condition.

Theorem 9 Let $\mathfrak{F}=\left\langle W,\left\langle\mathcal{N}_{i}\right\rangle_{i \in I}\right\rangle$ be a frame and let $w \in W$. Then $\mathcal{N}_{2}(w) \subseteq$ $\cap^{\infty} \mathcal{N}_{1}(w)$ iff $\mathfrak{F}, w \models \square_{2} p \rightarrow \square_{1 \infty} p$. Moreover, this frame condition cannot be characterized within $\mathfrak{L}_{f}^{[\forall]}$.

Proof Again, proving that the frame condition corresponds to the mentioned formula is left to the reader. To show that it cannot be characterized within $\mathfrak{L}_{f}^{[\forall]}$, let $I$ and $\mathfrak{M}$ be as in the proof of Theorem 8. Let $\mathfrak{M}^{\prime}=\left\langle W^{\prime},\left\langle\mathcal{N}_{i}^{\prime}\right\rangle_{i \in I}, V^{\prime}\right\rangle$ where $W^{\prime}=\left\{w_{i}^{\prime} \mid\right.$ $i \in \mathbb{N}\} \cup\left\{v^{\prime}\right\}, \mathcal{N}_{1}^{\prime}(x)=\left\{W^{\prime} \backslash\left\{w_{1}^{\prime}, \ldots, w_{n}^{\prime}\right\} \mid n \in\{1,2, \ldots\}\right\}$ for all $x \in W^{\prime}$, and $\mathcal{N}_{2}^{\prime}(x)=\left\{\left\{v^{\prime}\right\}\right\}$ for all $x \in W^{\prime} . V^{\prime}$ just treats each of the $w_{i}^{\prime}$ as copies of $w$ and $v^{\prime}$ as a copy of $v$. It can be easily verified that (a) no $X \in \mathcal{N}_{1}^{\prime}\left(w_{1}^{\prime}\right)$ is definable and (b) $\left\{v^{\prime}\right\} \in \Pi^{\infty} \mathcal{N}_{1}^{\prime}\left(w_{1}^{\prime}\right)$. From (b) it follows that $\mathfrak{F}^{\prime}=\left\langle W^{\prime},\left\langle\mathcal{N}_{i}^{\prime}\right\rangle_{i \in I}\right\rangle$ satisfies the frame condition of concern. Relying on (a) we can prove that $\left(\mathfrak{M}^{\prime}, w_{1}^{\prime}\right) \equiv_{\mathfrak{L}_{f}^{[\forall]}}(\mathfrak{M}, w)-\mathrm{a}$ contradiction.

Theorems 8 and 9 concern frame conditions that are relatively uncommon, though perhaps useful from the viewpoint of certain applications. After these warm-ups, we turn to a frame condition that is often discussed, i.e. closure under intersections. First, it is well-known that

$$
(\square p \wedge \square q) \rightarrow \square(p \wedge q)
$$

characterizes closure of the neighbourhood set under finite intersections - see e.g. [31, Lemma 2.20]. In contrast, there is no formula in $\mathfrak{L}^{[\forall]}$, or, for that matter, in $\mathfrak{L}_{f}^{[\forall]}$, that characterizes closure under arbitrary intersections. Here, infinitary pooling modalities turn out to yield a strict gain in expressive power, compared to the language with only finitary pooling modalities. Likewise, only with infinitary pooling modalities can one express that the intersection of all members of a neighbourhood is non-empty.

Theorem 10 Let $\mathfrak{F}=\left\langle W,\left\langle\mathcal{N}_{i}\right\rangle_{i \in I}\right\rangle$ be a frame and let $w \in W$. Then each of the following hold:

1. $\mathcal{N}_{i}(w)$ is closed under arbitrary intersections iff $\mathfrak{F}, w \models \square_{i} \infty p \rightarrow \square_{i} p$

2. $\bigcap \mathcal{N}_{i}(w) \neq \emptyset$ iff $\mathfrak{F}, w \models \square_{i} \infty p \rightarrow[\exists] p$.

Moreover, neither of these frame conditions can be characterized by formulas within $\mathfrak{L}_{f}^{[\forall]}$.

Proof Ad 1. Again, proving the positive claim is left to the reader. Although the negative claim for the language without $\square_{i} \infty$ modality is perhaps not too surprising, proving it requires some work. We provide a counterexample with $I=\{1\}$ and we omit the subscript when referring to the neighbourhoods for index 1 . Our counterexample uses constant neighbourhood functions, and neighbourhood sets that are 
closed under finite intersections. As a result, for every $k \in \mathbb{N}, \square_{1^{k}} \varphi$ is equivalent to $\square_{1}$ in the models we construct below. Hence, it suffices to focus on the basic modal language $\mathfrak{L}^{[\forall]}$ with a single operator $\square_{1}$.

Assume for contradiction that there is some $\varphi \in \mathfrak{L}^{[\forall]}$ that characterizes closure under arbitrary intersections. Let $W=\mathbb{N}$ and $W^{+}=\mathbb{N} \cup\{y\}$. Let, for all $n \in \mathbb{N}$, $X_{n}=W \backslash\{0, \ldots, n\}$ and $X_{n}^{+}=W^{+} \backslash\{0, \ldots, n\}$. Let $\mathcal{N}=\left\{X_{n} \mid n \in \mathbb{N}\right\}$ and $\mathcal{N}^{+}=\left\{X_{n}^{+} \mid n \in \mathbb{N}\right\} \cup\{\{y\}\}$. Let $\mathfrak{F}=\langle W, \mathcal{N}\rangle$ and $\mathfrak{F}^{+}=\left\langle W^{+}, \mathcal{N}^{+}\right\rangle$. These two frames are depicted in Fig. 6.

Note that $\mathcal{N}$ is not closed under arbitrary intersection, since it does not contain $\emptyset$, while $\bigcap \mathcal{N}=\emptyset$. By our assumption, there is a valuation $V$ on $W$ and a $v \in W$ such that $\langle\mathfrak{F}, V\rangle, v \models \neg \varphi$. In the remainder, let $\mathfrak{M}=\langle\mathfrak{F}, V\rangle$. So we have:

Fact $12 \mathfrak{M}, v \models \neg \varphi$.

We define the valuation $V^{+}: W^{+} \rightarrow \wp(\mathfrak{P})$ that extends $V$, as follows. First, for all $w \in W$, we let $V^{+}(w)=V(w)$. We define $V^{+}(y)$ inductively. Pick an enumeration $p_{1}, p_{2}, \ldots$ of $\mathfrak{P}$. As induction base let $\rho_{0}=\top$ and $\Delta_{0}=\emptyset$. Trivially, there are infinitely many $w \in W$ such that $\mathfrak{M}, w=\rho_{0}$. For the induction step assume that $\rho_{i-1}$ and $\Delta_{i-1}$ have already been defined, and there are infinitely many $w \in W$ such that $\mathfrak{M}, w \models \rho_{i-1}$. If there are infinitely many worlds $w$ such that $\mathfrak{M}, w=\rho_{i-1} \wedge p_{i}$, set $\rho_{i}=\rho_{i-1} \wedge p_{i}$ and $\Delta_{i}=\Delta_{i-1} \cup\left\{p_{i}\right\}$. Note that if, on the other hand, there are only finitely many worlds $w \in W$ such that $\mathfrak{M}, w \models \rho_{i-1} \wedge p_{i}$, then there are infinitely many worlds $w \in W$ such that $\mathfrak{M}, w \models \rho_{i-1} \wedge \neg p_{i}$. In the latter case, set $\rho_{i}=\rho_{i-1} \wedge \neg p_{i}$ and $\Delta_{i}=\Delta_{i-1}$. Finally, let $V^{+}(y)=\Delta_{0} \cup \Delta_{1} \cup \ldots$.

Let $\mathfrak{M}^{+}=\left\langle W^{+}, \mathcal{N}^{+}, V^{+}\right\rangle$. Note that $\mathcal{N}^{+}$is closed under arbitrary intersections. with $\bigcap_{X \in \mathcal{X}^{+}}=\{y\}$ for any infinite $\mathcal{X}^{+} \subseteq \mathcal{N}^{+}$. To arrive at a contradiction we prove that, for all worlds $w \in W,(\mathfrak{M}, w) \equiv_{\mathfrak{L}[\forall]}\left(\mathfrak{M}^{+}, w\right)$. This is Lemma 17 below; we first need a few other lemmata in preparation.

Lemma 13 For every $n, k \in \mathbb{N}$, there is a $w \in X_{n}$ such that $\mathfrak{M}^{+}, w \models \rho_{k}$.

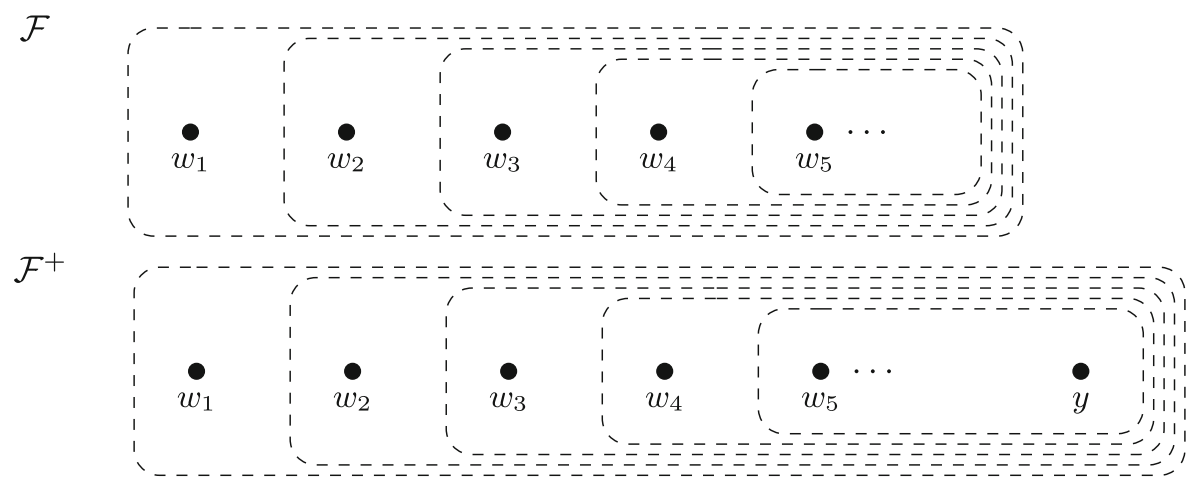

Fig. 6 The two frames $\mathcal{F}$ and $\mathcal{F}^{+}$in the proof of Theorem 10 
Proof Let $n, k \in \mathbb{N}$. By our construction, there are infinitely many $w \in W$ such that $\mathfrak{M}, w \models \rho_{k}$. Since $V^{+}$agrees with $V$ on all these $w$, and since $\rho_{k}$ is just a truth-functional compound of propositional variables, there are also infinitely many $w \in W$ such that $\mathfrak{M}^{+}, w \models \rho_{k}$. Since there are only finitely many $w \in W \backslash X_{n}$, it follows that there is at least one (in fact, there are infinitely many) $w \in X_{n}$ such that $\mathfrak{M}^{+}, w \models \rho_{k}$.

Lemma 14 For every finite set $\Delta$ of propositional variables, for every $n \in \mathbb{N}$ : there is a $w \in X_{n}$ such that, $V^{+}(w) \cap \Delta=V^{+}(y) \cap \Delta$.

Proof Let $\Delta$ be a finite set of propositional variables. Pick a $k \in \mathbb{N}$ such that all variables from $\Delta$ occur in $\rho_{k}$. Let $n$ be arbitrary. By the preceding lemma, there is a $w \in X_{n}$ such that $\mathfrak{M}^{+}, w \models \rho_{k}$. Note that also $\mathfrak{M}^{+}, y \models \rho_{k}$. In view of the construction of $\rho_{k}$, this means that $V^{+}(w) \cap \Delta=V^{+}(y) \cap \Delta$.

Lemma 15 For every $n \in \mathbb{N}$ and $\psi \in \mathfrak{L}^{[\forall]}$ : if, for all $w \in X_{n}$, $\mathfrak{M}^{+}, w \models \psi$, then $\mathfrak{M}^{+}, y \models \psi$.

Proof Suppose the antecedent holds. Since we work with constant neighbourhood functions, every formula of the form $\square \tau$ is either false everywhere or true everywhere in $\mathfrak{M}^{+}$. Hence, every such formula is either equivalent to $p \vee \neg p$ or to $p \wedge \neg p$ in $\mathfrak{M}^{+}$. The same applies mutatis mutandis to formulas of the form $[\forall] \tau$. As a result, there must be a truth-functional combination of propositional variables, say $\psi^{\prime}$, for which $\left\|\psi^{\prime}\right\|^{\mathfrak{M}^{+}}=\|\psi\|^{\mathfrak{M}^{+}}$. Let $\Delta$ be the (finite) set of variables that occur in $\psi^{\prime}$. Note that the truth value of $\psi^{\prime}$ at any world $w \in W^{+}$is a function of $V^{+}(w) \cap \Delta$. By Lemma 14 , there is a $w \in X_{n}$ such that $V^{+}(w) \cap \Delta=V^{+}(y) \cap \Delta$. Consequently, $\mathfrak{M}^{+}, y \models \psi^{\prime}$ and hence $\mathfrak{M}^{+}, y=\psi$.

Corollary 3 For every $n \in \mathbb{N}$ and $\psi \in \mathfrak{L}^{[\forall]}$ : if $\mathfrak{M}^{+}, y=\psi$, then there is a $w \in X_{n}$ such that $\mathfrak{M}^{+}, w \models \psi$.

Corollary 4 For every $\psi \in \mathfrak{L}^{[\forall]}$ : if $\mathfrak{M}^{+}, y \models \psi$, then there is a $w \in W$ such that $\mathfrak{M}^{+}, w \models \psi$.

Lemma 16 For every $\psi \in \mathfrak{L}^{[\forall]}$, each of the following hold:

1. $\mathfrak{M}, w \models \square \psi$ iff there is an $n \in \mathbb{N}$ such that $X_{n}=\|\psi\|^{\mathfrak{M}}$

2. $\mathfrak{M}^{+}, w \models \square \psi$ iff there is an $n \in \mathbb{N}$ such that $X_{n}^{+}=\|\psi\|^{\mathfrak{M}^{+}}$

Proof Item (1.) is immediate in view of the definition of $\mathcal{N}$. For item (2.) it suffices to prove that there is no $\psi \in \mathfrak{L}_{f}^{[\forall]}$ such that $\|\psi\|^{\mathfrak{M}^{+}}=\{y\}$. So assume there is one such $\psi$. Then $\mathfrak{M}^{+}, y \models \psi$. By Corollary 4, there is a $w \in W$ such that $\mathfrak{M}^{+}, w \models \psi$. But then $w \in\|\psi\|^{\mathfrak{M}^{+}}$- a contradiction, since $w \neq y$.

Lemma 17 For all $w \in W$ and all $\psi \in \mathfrak{L}_{f}^{[\forall]}$, each of the following hold:

(i) $\mathfrak{M}, w \models \psi$ iff $\mathfrak{M}^{+}, w \models \psi$ 
(ii) $\|\psi\|^{\mathfrak{M}}=\|\psi\|^{\mathfrak{M}^{+}} \cap W$

(iii) for all $n \in \mathbb{N}, X_{n}=\|\psi\|^{\mathfrak{M}}$ iff $X_{n}^{+}=\|\psi\|^{\mathfrak{M}^{+}}$

Proof (i) and (ii) are easily seen to be equivalent. We prove (i)/(ii) and (iii) by a simultaneous induction on the complexity of $\psi$. The base case $(\psi \in \mathfrak{P})$ for (i)/(ii) is immediate in view of the construction. For (iii), we start with right to left. Suppose $X_{n}^{+}=\|\psi\|^{\mathfrak{M}^{+}}$. Then $X_{n}^{+} \cap W=\|\psi\|^{\mathfrak{M}^{+}} \cap W$. Since $X_{n}=X_{n}^{+} \cap W$ and by (ii), $X_{n}=\|\psi\|^{\mathfrak{M}}$. For left to right, suppose that $X_{n}=\|\psi\|^{\mathfrak{M}}$. So for all $w \in X_{n}$, $\mathfrak{M}, w \models \psi$. By (i), for all $w \in X_{n}, \mathfrak{M}^{+}, w \models \psi$. By Lemma 15, $\mathfrak{M}^{+}, y \models \psi$. By (ii), $\|\psi\|^{\mathfrak{M}^{+}} \cap W=\|\psi\|^{\mathfrak{M}}=X_{n}$. Hence, $X_{n}^{+}=X_{n} \cup\{y\}=\|\psi\|^{\mathfrak{M}^{+}}$.

For the induction step for (i)/(ii), we only need to consider formulas of the form $\square_{1} \psi$ (cf. our remark at the outset of the proof of Theorem 10). We have: $\mathfrak{M}, w \models$ $\square_{1} \psi$ iff [by Lemma 16.1] there is some $n \in \mathbb{N}$ such that $X_{n}=\|\psi\|^{\mathfrak{M}}$ iff [by the induction hypothesis, item (iii)] there is some $n \in \mathbb{N}$ such that $X_{n}^{+}=\|\psi\|^{\mathfrak{M}^{+}}$iff [by Lemma 16.2] $\mathfrak{M}^{+}, w \models \square_{1} \psi$.

For (iii), we reason just as for the base case, relying on the fact that (i)/(ii) was already proven for $\psi$.

By Fact 12 and Lemma 17.(i), $\mathfrak{M}^{+}, v \models \neg \varphi$, contradicting the assumption that $\varphi$ characterizes closure under arbitrary intersections. This completes our proof of item 1 of Theorem 10 .

Ad 2. The positive claim is again safely left to the reader. The negative claim follows from our proof for item 1 of Theorem 10: in the above construction, $\bigcap \mathcal{N}=$ $\emptyset$, whereas $\bigcap \mathcal{N}^{+}=\{y\}$.

\section{Pooling Dynamics}

In the preceding, we focused on static modal operators that express what sets can be obtained by pointwise intersections of neighbourhood collections. However, the term "pooling" is also often associated with dynamics of multi-agent systems. The aim of this section is to provide a rough introduction to such pooling dynamics in the context of neighbourhood semantics, and to show the use of static pooling modalities in studying such dynamics. We first go over a number of concrete examples of pooling dynamics in informal terms (Sections 6.1 and 6.2). Next, we spell out a generic format of model updates that covers each of these examples (Section 6.3), and give a complete set of reduction axioms for all updates in this format (Section 6.4).

\subsection{Pooling Information}

In the context of normal S5-type modalities for knowledge, Ågotnes and Wáng have introduced so-called resolution operators for multi-agent epistemic logics [1]. These operators allow one to speak about the updated model in which some of the agents have exchanged all their knowledge with each other (while it is publically observed that this event happens). More formally, given a multi-agent epistemic model 
$\mathfrak{M}=\left\langle W,\left\langle\sim_{i}\right\rangle_{i \in I}, V\right\rangle$ and a finite group $G$, the resolution operator $R_{G}$ allows to express what holds in the model $\mathfrak{M}_{G}^{\prime}=\left\langle W,\left\langle\sim_{i}^{\prime}\right\rangle_{i \in I}, V\right\rangle$ where

(R1) $\quad \sim_{i}^{\prime}=\sim_{i}$ if $i \notin G$

(R2) $\quad \sim_{i}^{\prime}=\bigcap_{j \in G} \sim_{j}$ if $i \in G$

The semantics of the dynamic operator $R_{G}$ is provided by the following clause:

$$
\mathfrak{M}, w \vDash R_{G} \varphi \text { iff } \mathfrak{M}_{G}^{\prime}, w \vDash \varphi,
$$

In what follows, we show how various types of resolution and, more generally, informational dynamics across agents, can be defined and distinguished in the context of neighbourhood semantics. To fix intuitions, we interpret neighbourhoods in terms of (fallible, possibly conflicting) evidence of the agents $i \in I$. That is, $X \in \mathcal{N}_{i}(w)$ means that at world $w$, agent $i$ has $X$ among its evidence. Accordingly, $\square_{i} \varphi$ can be interpreted as "agent $i$ has (the) evidence that $\varphi$ ". ${ }^{26}$

When different agents have distinct evidence, it makes sense to ask what becomes true if those agents communicate about their evidence. In what follows, we focus on cases where some agents share all their information with other agents at every world $w$ in the model. In other words, the dynamics we will discuss are cases of complete, global pooling. ${ }^{27}$

These updates are defined by specifying what each updated neighbourhood collection $\mathcal{N}_{i}^{\prime}(w)$ looks like, given the original tuple $\left\langle\mathcal{N}_{i}(w)\right\rangle_{i \in I}$. We will discuss various such updates in the present subsection, focusing on the case of two agents $i$ and $j$; all our observations generalize easily to larger groups.

A first important distinction concerns the direction of communication. In particular, agent $j$ may receive evidence from agent $i$ without $j$ providing any evidence to $i$. This is distinct from the case - analogous to the aforementioned resolution operators - in which all involved agents exchange all their information with each other. We call the former type of pooling asymmetric, and the latter symmetric. Second, we can ask whether $j$ merely adds pieces of evidence of $i$ to its former collection of evidence, or whether instead $j$ also processes the newly obtained evidence by intersecting it with its former evidence. Formally, this boils down to the distinction between the following two asymmetric pooling types: weak asymmetric pooling from $i$ to $j$ :

$$
\mathcal{N}_{j}^{\prime}(w)=\mathcal{N}_{j}(w) \cup \mathcal{N}_{i}(w) \text { and } \mathcal{N}_{i}^{\prime}(w)=\mathcal{N}_{i}(w)
$$

\footnotetext{
${ }^{26}$ This interpretation follows [40], except that we focus on "having evidence that", whereas van Benthem and Pacuit use the monotonic semantic clause (cf. Section 4.3), and interpret the modal operator in terms of "having evidence for". We do not presuppose that evidence is truthful, closed under conjunction, or closed under logical consequence. In fact, in most of what follows and unless stated otherwise, we assume no conditions on the neighbourhood functions. In the literature on evidence logic, it is typically assumed that $W \in \mathcal{N}_{i}(w)$ and that $\emptyset \notin \mathcal{N}_{i}(w)$, cf. [40]. Often it is also assumed that $\mathcal{N}_{i}$ is uniform, in the sense that for all $w, w^{\prime}, \mathcal{N}_{i}(w)=\mathcal{N}_{i}\left(w^{\prime}\right)$, cf. [3].

${ }^{27}$ In this sense we stick to the format of the resolution operators in [1]. Examples of partial, global information update in a single-agent, non-normal setting can be found in [40], interpreted as the public announcement, addition, removal, or modification of specific pieces of evidence. While there are some links with the logic of private announcements $[4,46]$, we know of no work that tackles truly local updates, in the sense that only the neighbourhood collection of a single world is changed. Such updates would in any case not be expressible in the languages from this paper.
} 
and strong asymmetric pooling from $i$ to $j$ :

$$
\mathcal{N}_{j}^{\prime}(w)=\mathcal{N}_{j}(w) \cap \mathcal{N}_{i}(w) \text { and } \mathcal{N}_{i}^{\prime}(w)=\mathcal{N}_{i}(w)
$$

Since we make no assumptions about the neighbourhood collections, strong asymmetric pooling may lead to a loss of information - this will e.g. be the case for agent $i$ if $\mathcal{N}_{j}(w)=\emptyset$ (but $\mathcal{N}_{i}(w) \neq \emptyset$ ) or more generally, if not every member of $\mathcal{N}_{i}(w)$ is included in some member of $\mathcal{N}_{j}(w)$. If, on the other hand, each neighbourhood collection contains the unit $W$, then such information loss does not arise and strong pooling is by definition conservative. This assumption is natural for various interpretations of neighbourhood semantics, though we do not build it in here for the sake of generality. ${ }^{28}$

If said assumption cannot be built in, one may still consider strong, conservative asymmetric pooling from $i$ to $j$ :

$$
\mathcal{N}_{j}^{\prime}(w)=\left(\mathcal{N}_{j}(w) \cap \mathcal{N}_{i}(w)\right) \cup \mathcal{N}_{j}(w) \cup \mathcal{N}_{i}(w) \text { and } \mathcal{N}_{i}^{\prime}(w)=\mathcal{N}_{i}(w)
$$

Turning to symmetric pooling, we can again distinguish between a weak and a strong variant. For the binary case, weak symmetric pooling among $i$ and $j$ is given by:

$$
\mathcal{N}_{i}^{\prime}(w)=\mathcal{N}_{i}(w) \cup \mathcal{N}_{j}(w) \text { and } \mathcal{N}_{j}^{\prime}(w)=\mathcal{N}_{i}(w) \cup \mathcal{N}_{j}(w)
$$

whereas strong symmetric pooling among $i$ and $j$ boils down to:

$$
\mathcal{N}_{i}^{\prime}(w)=\mathcal{N}_{i}(w) \cap \mathcal{N}_{j}(w) \text { and } \mathcal{N}_{j}^{\prime}(w)=\mathcal{N}_{i}(w) \cap \mathcal{N}_{j}(w)
$$

As before, one can also define a conservative variant of strong symmetric pooling, which will arguably be non-equivalent with the non-conservative variant just defined over the general class of neighbourhood models.

One may also consider the case where an agent performs self-pooling, i.e. intersects its own pieces of evidence. This can be characterized as strong (symmetric or asymmetric) pooling of an agent $i$ with itself. Or alternatively, we may consider more complicated dynamics in which $i$ intersects its own evidence and combines this with new evidence obtained from another agent. And so on - we have merely scratched the surface of logical possibilities here. Having spelled out basic pooling operations such as the above, one can start investigating combinations or iterations of them, giving rise to an algebra of pooling operations. Our point here was merely to show the diversity of pooling operations and their interpretations; we believe this calls for a unifying formal approach and will develop that in Sections 6.3 and 6.4. First, however, we turn to a yet different type of pooling dynamics.

\subsection{Power Dynamics}

In logics of abilities or strategic powers, $X \in \mathcal{N}_{i}(w)$ can be interpreted as the agent having some available action that brings about $X$. Here, closure under finite

\footnotetext{
${ }^{28}$ In the companion paper [43] we provide sound and strongly complete axiomatizations for various logics with and without this extra assumption.

In view of the reduction axioms provided below, we also obtain axiomatizations for extension of those logics with the dynamic operators introduced in this section.
} 
intersections - condition $\left(\mathrm{C}_{i}\right)$ in Table 1 - need not hold, as an agent's option to choose between various possible actions does not imply that she can execute several of these simultaneously. Moreover, logics of exact strategic abilities invalidate necessitation $\left(\mathrm{N}_{i}\right)$ and monotony $\left(\mathrm{M}_{i}\right)$. In [37] such logics are shown to be instrumental for defining notions of equivalence between games.

In this context, dynamic operations also naturally arise. For instance, one may ask what becomes true if an agent $i$ delegates its powers to another agent $j .{ }^{29}$ One candidate explication of this idea would be given by the following equations:

$$
\mathcal{N}_{i}^{\prime}(w)=\emptyset \text { and } \mathcal{N}_{j}^{\prime}(w)=\mathcal{N}_{j}(w) \cap \mathcal{N}_{i}(w)
$$

Let us call this delegation from $i$ to $j$. Intuitively, it tells us that $j$ ends up with the powers of the group $\{i, j\}$ after $i$ has delegated to $j$. Although seemingly intuitive, it has the strange property that if either $i$ or $j$ start off having no powers at all, then also $\mathcal{N}_{j}^{\prime}(w)=\emptyset$. (Note that $\emptyset \cap \mathcal{X}=\varnothing$ for all $\mathcal{X}$.) One may therefore consider a slightly different explication, which - in keeping with Section 6.1 - could be called conservative delegation from $i$ to $j$ :

$$
\mathcal{N}_{i}^{\prime}(w)=\emptyset \text { and } \mathcal{N}_{j}^{\prime}(w)=\left(\mathcal{N}_{j}(w) \cap \mathcal{N}_{i}(w)\right) \cup \mathcal{N}_{i}(w) \cup \mathcal{N}_{j}(w)
$$

Note that, unlike the cases of information dynamics considered above, $i$ delegating to $j$ implies that $i$ necessarily loses all its powers (if any), and ends up with an empty neighbourhood collection. ${ }^{30}$ The counterpart of this behavior for epistemicdoxastic interpretations would be an event where one agent "passess on" its evidence to another agent, and afterwards completely loses track of that evidence.

Having specified relatively simple updates associated with power delegations, one can ask how complex patterns of delegation behave. One central case is a delegation cycle where $i$ delegates to $j, j$ to $k$, and $k$ again to $i$ [6]. If we apply the above definitions to such a cycle using the non-conservative variant, then all three agents will end up having no powers: $\mathcal{N}_{i}^{\prime \prime \prime}(w)=\mathcal{N}_{j}^{\prime \prime \prime}(w)=\mathcal{N}_{k}^{\prime \prime \prime}(w)=\emptyset$. In contrast, in the conservative variant, agent $i$ will end up accumulating all powers of itself, $j$, and $k$, whereas $i$ and $j$ are left powerless. If one finds neither outcomes attractive, one may define alternative formats of combined or sequential delegation that are sensitive to cycles - e.g. by giving all agents back their original powers when they are in a delegation cycle.

We leave it there for our outline of different pooling dynamics. We are convinced that each of these, and many more, deserve further study in their own right. In what

\footnotetext{
${ }^{29}$ One natural context where such delegations occur is Liquid Democracy, a voting system where people can either vote themselves or delegate their voting power to others [5, 11]. See also [45] for extensions of coalition logic with dynamic operators for delegation. Note that, whereas Van der Hoek and Wooldridge [45] consider the delegation of specific powers (to change the value of specified variables), we here focus on one agent delegating all its powers to another agent, cf. our earlier distinction between partial and complete pooling dynamics.

${ }^{30}$ Alternatively, one may stipulate that the unit $W$ is the only power that is left for $i$ after delegating to $j$, so that $\mathcal{N}_{i}^{\prime}(w)=\{W\}$. This would match better with the standard approach in coalition logic, where it is required that for all models and all agents, the unit is contained in the agent's abilities. Syntactically this means that $\square_{i} \top$ is valid.
} 
follows, we will however focus on the possibility of expressing them using pooling modalities.

\subsection{A Generic Format for Updates...}

The following definition specifies the class of all updates that are focal in this section:

Definition 14 A profile update is any function $f: I \rightarrow \wp\left(\mathbb{M}_{\infty}\right)$ where, for all $i \in I, f(i)$ is a finite subset of $\mathbb{M}_{\infty}$.

Roughly speaking, a profile update specifies the neighbourhood collection of each agent after the update took place, in terms of the neighbourhood collections of specific pooling profiles before the update took place. For instance, that $\{(i, 1),(j, 1)\} \in$ $f(i)$ could be interpreted as: "any information obtained by intersecting evidence of $i$ and $j$ becomes evidence of $i$ after the update" (cf. Section 6.1). The update of a given model is defined accordingly:

Definition 15 Let $\mathfrak{M}=\left\langle W,\left\langle\mathcal{N}_{i}\right\rangle_{i \in I}, V\right\rangle$ and let $f$ be a profile update. The update of $\mathfrak{M}$ with $f$ is given by $\mathfrak{M}^{f}=\left\langle W,\left\langle\mathcal{N}_{i}^{f}\right\rangle_{i \in I}, V\right\rangle$, where for all $i \in I$ and all $w \in W$,

$$
\mathcal{N}_{i}^{f}(w)=\bigcup_{M \in f(i)} \mathcal{N}_{M}(w)
$$

Table 3 illustrates the notion of a profile update in terms of the examples we discussed previously. Note that, as shown by the case for delegation, it is possible for a profile update $f$ to map a given index $i$ to the empty set. In this case, it can be readily verified that, for every $K$ with $i \in I(K), \mathcal{N}_{K}(w)=\emptyset$ as well.

In what follows we will say that a profile update $f$ is finitary if and only if its codomain is $\wp\left(\mathbb{M}_{f}\right) ; f$ is simple if and only if for every $i \in I$, for every $M \in f(i)$, $M$ is of the type $\{(j, 1)\} \cup\{(k, 0) \mid k \neq j\}$ for some $j \in I$. The weak pooling types mentioned in Section 6.1 are simple, whereas the strong and conservative ones are not simple but still finitary. An example of an update that is not finitary would be one where some or all agents close all their neighbourhood collections under arbitrary intersections; this is given by $f(i)=\{(i, \infty)\}$ (for some or all $i \in I) .^{31}$

At the syntactic level, we can define dynamic operators $[f]$ for every corresponding profile update $f$. We interpret these in the expected way:

Definition 16 Let $\mathfrak{M}=\left\langle W,\left\langle\mathcal{N}_{i}\right\rangle_{i \in I}, V\right\rangle$, let $f$ be a profile update, and let $\varphi$ be a formula in the language enriched with dynamic pooling operators. Then, for every $w \in W$ :

$$
\mathfrak{M}, w \models[f] \varphi \text { iff } \mathfrak{M}^{f}, w \models \varphi \text {. }
$$

\footnotetext{
${ }^{31}$ An operation of this type is studied (for the single-agent case) in [40, Section 4.5] under the header of evidence combination.
} 
Table 3 Update profiles that correspond the various types of dynamics mentioned in Sections 6.1 and 6.2. For all $k \neq i, j$, we have $f(k)=\{(k, 1)\}$

\begin{tabular}{lll}
\hline information pooling & $f(i)=$ & $f(j)=$ \\
\hline asymmetric, from $i$ to $j$ & $\{\{i\}\}$ & \\
weak & $\{\{i\}\}$ & $\{\{i\},\{j\}\}$ \\
strong & $\{\{i\}\}$ & $\{\{i, j\}\}$ \\
conservative, strong & & $\{\{j\},\{i\},\{i, j\}\}$ \\
symmetric, among $i$ and $j$ & $\{\{i\},\{j\}\}$ & \\
weak & $\{\{i, j\}\}$ & $\{\{i\},\{j\}\}$ \\
strong & $\{\{i\},\{j\},\{i, j\}\}$ & $\{\{i, j\}\}$ \\
conservative, strong & & $\{\{i\},\{j\},\{i, j\}\}$ \\
power delegation, from $i$ to $j$ & $\emptyset$ & $\{\{i, j\}\}$ \\
non-conservative & $\emptyset$ & $\{\{i\},\{j\},\{i, j\}\}$ \\
conservative & &
\end{tabular}

Thus, letting $f_{i \rightarrow j}$ (resp. $f_{i \Rightarrow j}$ ) stand for weak (strong) asymmetric pooling from $i$ to $j$, the formulas $\neg \square_{j} p \wedge\left[f_{i \rightarrow j}\right] \square_{j} p$ resp. $\neg \square_{j} p \wedge\left[f_{i \Rightarrow j}\right] \square_{j} p$ express that after such pooling, $j$ has the evidence that $p$, while it did not have such evidence before. Letting $f_{i \Leftrightarrow j}$ stand for strong symmetric pooling, a formula such as

$$
\left[f_{i \Leftrightarrow j}\right] \square_{j} \infty \varphi \rightarrow\left[f_{i \rightarrow j}\right] \square_{j} \infty \varphi
$$

can be used to express that any (implicit) information $j$ has after symmetric pooling with $i$ will already be had by $j$ after weak asymmetric pooling from $i$ to $j$.

\section{4 ... and its Reduction Axioms}

The extension of our four base languages with dynamic operators $[f]$ is straightforward. To axiomatize the resulting logics, we use the familiar technique of providing reduction axioms, which essentially show that all formulas in the dynamic language are equivalent to formulas in the original base language [4]. Recall that a sound and complete axiomatization of the base logics is given in [43]. Together with the reduction axioms that we discuss below, this also gives us a sound and strongly complete axiomatization for the extended languages that feature dynamic operators.

The reduction axioms for the connectives and $[\forall]$ work exactly as expected (cf. axioms R1-R4 in Table 4). For the (static) pooling modalities, (R5) expresses that after updating with $f$, the extension of $\varphi$ (in the updated model) is in $\mathcal{N}_{M}$ if and only if the extension of $[f] \varphi$ in the original model (and hence, again, the extension of $\varphi$ in the updated model) is contained in some neighbourhood $\mathcal{N}_{K}$, where $K$ ranges over a well-defined (finite) set of pooling profiles $\Omega_{M}^{f}$ that depends on both $f$ and $M$.

Before we turn to the definition of that set, let us try to illustrate what is going on. Recall that a pooling profile $M$ can be rewritten as a (possibly infinite) multiset, such 
Table 4 Reduction axioms for dynamic operators $[f]$

\begin{tabular}{ll}
\hline (R1) & {$[f] \varphi \leftrightarrow \varphi$ for every $\varphi \in \mathfrak{P}$} \\
(R2) & {$[f] \neg \varphi \leftrightarrow \neg[f] \varphi$} \\
(R3) & {$[f](\varphi \vee \psi) \leftrightarrow([f] \varphi \vee[f] \psi)$} \\
(R4) & {$[f][\forall] \varphi \leftrightarrow[\forall][f] \varphi$} \\
(R5) & {$[f] \square_{M} \varphi \leftrightarrow \bigvee_{K \in \Omega_{M}^{f}} \square_{K}[f] \varphi$} \\
\hline
\end{tabular}

Recall that, in case $\Delta=\emptyset, \bigvee \Delta=\perp$. Hence, for cases where $\Omega_{M}^{f}=\emptyset$, axiom (R5) reads: $[f] \square_{M} \varphi \leftrightarrow \perp$

as e.g. $M=\left\{1,1,2,2,2,3,4^{\infty}\right\}$. Then the neighborhood $\mathcal{N}_{M}$ in the updated model $\mathfrak{M}^{f}$ is equivalent to a union of various neighborhoods $\mathcal{N}_{K}$ of $\mathfrak{M}$, where each such $K$ can be obtained by substituting each occurrence of an $i$ in the multiset $M$ with some member $N$ of $f(i)$. Notably, different occurrences of the same index $i$ in $M$ may be replaced by different $N \in F(i)$. Take the earlier example with $f(2)=\left\{N_{1}, N_{2}, N_{3}\right\}$. When evaluating a formula like $\square_{\{2,2,2\}} p$ after the update with $f$, we may replace the index 2 once with $N_{1}$ and twice with $N_{2}$.

To spell this out in full generality, we first need some extra notation. First, for pooling profiles $M$ and $M^{\prime}$ we use $M \sqcup M^{\prime}$ to denote the union of these pooling profiles, i.e. the function $I \rightarrow \mathbb{N} \cup\{\infty\}$ sending $i$ to $M(i)+M^{\prime}(i)$. Clearly, $M \sqcup M^{\prime}$ is again a pooling profile. Second, for $k \in \mathbb{N}^{+} \cup\{\infty\}$ and $M$ a pooling profile, we let $k \cdot M$ be the pooling profile sending each $i \in I$ to $k \cdot M(i)$. Furthermore, we assume the standard property that, for all $k \in \mathbb{N} \cup\{\infty\}, k \cdot \infty=k+\infty=\infty$.

Definition 17 Let $M \in \mathbb{M}_{\infty}$ and let $f$ be a profile update. For every $i \in I(M)$, let

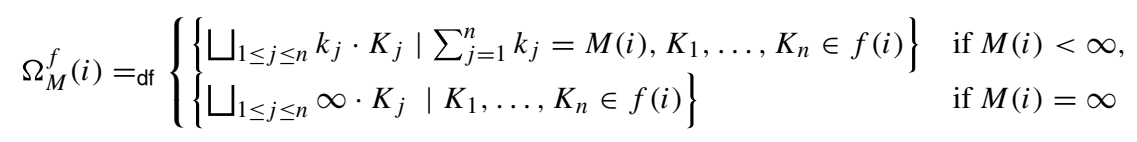

Finally, let $\Omega_{M}^{f}=_{\mathrm{df}}\left\{\bigsqcup_{i \in I_{M}} K_{i} \mid K_{i} \in \Omega_{M}^{f}(i)\right\}$.

Note that, for every $i \in I(M), \Omega_{M}^{f}(i)$ is a finite set of multisets. Intuitively, the members of $\Omega_{M}^{f}(i)$ can be obtained by replacing all occurrences of $i$ in $M$ with some $K \in f(i) .{ }^{32}$ Since $I(M)$ and all $f(i)$ are finite, $\Omega_{M}^{f}$ is a finite set.

\footnotetext{
${ }^{32}$ To keep $\Omega_{M}^{f}(i)$ finite, it does not contain all possible ways of replacing occurrences of $i$ with elements of $f(i)$. Rather, for those $i$ with $M(i)=\infty, \Omega_{M}^{f}(i)$ only consists of those replacements where each $K \in f(i)$ is either used infinitely often or not at all. Lemma 21 shows that this restriction is harmless.
} 
The following theorem is the key to the validity of (R5):

Theorem 11 Let $\mathfrak{M}=\left\langle W,\left\langle\mathcal{N}_{i}\right\rangle_{i \in I}, V\right\rangle$, let $f$ be a profile update, and let $M \in \mathbb{M}_{\infty}$. Let $\mathcal{N}_{M}^{f}$ denote the neighbourhood set of $M$ in the model $\mathfrak{M}^{f}$ and let $w \in W$.We have:

$$
\mathcal{N}_{M}^{f}(w)=\bigcup_{K \in \Omega_{M}^{f}} \mathcal{N}_{K}(w)
$$

Proof The proof requires quite some bookkeeping, in turn explaining our notational conventions leading up to Definition 17 . We start by noting a number of facts, then prove two lemmas, and finally tackle the theorem itself.

Fact 18 Let $\mathcal{X}$ be a set of sets and let $l \leq k \in \mathbb{N}$. Then $\cap^{l} \mathcal{X} \subseteq \cap^{k} \mathcal{X} \subseteq \cap^{\infty} \mathcal{X}$.

Fact 19 For every $K_{1}, \ldots, K_{n} \in \mathbb{M}_{\infty}, \mathcal{N}_{K_{1}}(w) \cap \ldots$ ก $\mathcal{N}_{K_{n}}(w)=\mathcal{N}_{K_{1} \sqcup \ldots \sqcup K_{n}}(w)$.

As $k \cdot K=\sqcup_{i \leq k} K$ for $k \in \mathbb{N} \cup\{\infty\}$, this entails that

Fact 20 For every $K \in \mathbb{M}_{\infty}$ and $k \in \mathbb{N} \cup\{\infty\}$, $\cap^{k} \mathcal{N}_{K}(w)=\mathcal{N}_{k \cdot K}(w)$.

Relying on the preceding facts, we can show a slightly more intricate property:

Lemma 21 For any finite $\mathcal{K} \subseteq \mathbb{M}_{\infty}$ and $X \subseteq W$, the following two statements are equivalent:

(1) there are $k_{1}, \ldots, k_{n}$ with $k_{1}+\ldots+k_{n}=\infty$ and $K_{1}, \ldots, K_{n} \in \mathcal{K}$ such that $X \in \mathcal{N}_{\bigsqcup_{1 \leq j \leq n} k_{j} \cdot K_{j}}(w)$

(2) there are $K_{1}^{\prime}, \ldots, K_{m}^{\prime} \in \mathcal{K}$ such that $X \in \mathcal{N}_{\bigsqcup_{1 \leq j \leq n} \infty \cdot K_{j}}(w)$

Proof By Facts 19 and 20, we can rewrite (1) and (2) as follows:

(1') there are $k_{1}, \ldots, k_{n}$ with $k_{1}+\ldots+k_{n}=\infty$ and $K_{1}, \ldots, K_{n} \in \mathcal{K}$ such that $X \in \cap^{k_{1}} \mathcal{N}_{K_{1}}(w) \cap \ldots \cap \cap^{k_{n}} \mathcal{N}_{K_{n}}(w)$

(2') there are $K_{1}^{\prime}, \ldots, K_{m}^{\prime} \in \mathcal{K}$ such that $X \in \cap^{\infty} \mathcal{N}_{K_{1}^{\prime}}(w) \cap \ldots \cap \cap^{\infty} \mathcal{N}_{K_{m}^{\prime}}(w)$

The implication from (2') to (1') is immediate. The implication from (1') to (2') follows from Fact 18.

Lemma 22 Let $M$ be a pooling profile and $f$ be a profile update. For all $i \in I(M)$ :

$$
X \in \bigcup_{N \in \Omega_{M}^{f}(i)} \mathcal{N}_{N}(w) \text { iff } \exists \mathcal{Y} \subseteq \mathcal{N}_{i}^{f}(w) \text { with }|\mathcal{Y}| \leq M(i) \text { such that } X=\bigcap_{Y \in \mathcal{Y}} Y
$$


Proof Let $i \in I(M)$. We observe a series of equivalences:

$$
\exists \mathcal{Y} \subseteq \mathcal{N}_{i}^{f}(w) \text { with }|\mathcal{Y}| \leq M(i) \text { such that } X=\bigcap_{Y \in \mathcal{Y}} Y
$$

$\stackrel{\text { Def. } 15}{\Longleftrightarrow} \exists k_{1}, \ldots, k_{n}$ with $k_{1}+\ldots+k_{n}=M(i), K_{1}, \ldots, K_{n} \in f(i)$,

and $\mathcal{Y}_{1}, \ldots, \mathcal{Y}_{n}$ with $\left|\mathcal{Y}_{j}\right| \leq k_{j}$ and $\mathcal{Y}_{j} \subseteq \mathcal{N}_{K_{j}}(w)$ for each $j \leq k$

$$
\text { such that } X=\bigcap_{1 \leq i \leq n} \bigcap_{Y \in \mathcal{Y}_{i}} Y
$$

Def. 5, Fact 18

$\exists k_{1}, \ldots, k_{n}$ with $k_{1}+\ldots+k_{n}=M(i)$ and $K_{1}, \ldots, K_{n} \in f(i)$

such that $X \in \cap^{k_{1}} \mathcal{N}_{K_{1}}(w) \cap \ldots \cap \cap^{k_{n}} \mathcal{N}_{K_{n}}(w)$

$\stackrel{\text { Facts } 19}{\Longleftrightarrow}, 20 \exists k_{1}, \ldots, k_{n}$ with $k_{1}+\ldots+k_{n}=M(i)$ and $K_{1}, \ldots, K_{n} \in f(i)$

such that $X \in \mathcal{N}_{\bigsqcup_{1 \leq j \leq n} k_{j} \cdot K_{j}}(w)$

Def. $\stackrel{17, \text { Lem. }}{\Longleftrightarrow} 21$

$$
X \in \bigcup_{N \in \Omega_{M}^{f}(i)} \mathcal{N}_{N}(w)
$$

The proof of Theorem 11 itself again proceeds in terms of a series of equivalences:

$$
\begin{array}{ll} 
& X \in \mathcal{N}_{M}^{f}(w) \\
\stackrel{\text { Def. . }}{\Longleftrightarrow} & \exists\left\langle\mathcal{Y}_{i} \subseteq \mathcal{N}_{i}^{f}(w)|i \in I(M),| \mathcal{Y}_{i} \mid \leq M(i)\right\rangle \text { such that } \bigcap_{i \in I(M)} \bigcap_{i} \mathcal{Y}_{i}=X \\
\stackrel{\text { Lem. 22 }}{\Longleftrightarrow} & X \in \cap_{i \in I(M)} \bigcup_{N \in \Omega_{M}^{f}(i)} \mathcal{N}_{N}(w) \\
& \stackrel{\text { Def. 5 }}{\Longleftrightarrow} \exists\left\langle K_{i} \mid i \in I(M), K_{i} \in \Omega_{M}^{f}(i)\right\rangle \text { such that } X \in \cap_{i \in I(M)} \mathcal{N}_{K_{i}}(w) \\
\stackrel{\text { Fact 19 }}{\Longleftrightarrow} \exists\left\langle K_{i} \mid i \in I(M), K_{i} \in \Omega_{M}^{f}(i)\right\rangle \text { such that } X \in \mathcal{N}_{\sqcup_{i \in I(M)} K_{i}}(w) \\
\stackrel{\text { Def. 17 }}{\Longleftrightarrow} \quad X \in \bigcup_{K \in \Omega_{M}^{f}} \mathcal{N}_{K}(w)
\end{array}
$$

Theorem 12 The reduction axioms (R1)-(R5) of Table 4 are valid on the class of neighborhood models.

Proof We show how the proof works for (R4) and (R5); the proofs of the other reduction axioms are analogous but simpler, and hence safely left to the reader.

Ad (R4). We have: $\mathfrak{M}, w \models[f][\forall] \varphi$ iff [by Definition 16] $\mathfrak{M}^{f}, w \models[\forall] \varphi$ iff [by Definition 2.5] for every $w^{\prime} \in W, \mathfrak{M}^{f}, w^{\prime} \models \varphi$ iff [by Definition 16] for every $w^{\prime} \in W, \mathfrak{M}, w^{\prime} \models[f] \varphi$ iff [by Definition 2.5] $\mathfrak{M}, w \models[\forall][f] \varphi$.

Ad (R5)] We have: $\mathfrak{M}, w \models[f] \square_{M} \varphi$ iff [by Definition 16] $\mathfrak{M}^{f}, w \models \square_{M} \varphi$ iff [by Definition 8] $\|\varphi\|^{\mathfrak{M}^{f}} \in \mathcal{N}_{M}^{f}(w)$ iff [by Definition 16] $\|[f] \varphi\|^{\mathfrak{M}} \in \mathcal{N}_{M}^{f}(w)$ iff 
[by Theorem 11] $\|[f] \varphi\|^{\mathfrak{M}} \in \cup_{K \in \Omega_{M}^{f}} \mathcal{N}_{K}(w)$ iff [by Definition 8 and Definition 2.3] $\mathfrak{M}, w \models \bigvee_{K \in \Omega_{M}^{f}} \square_{K}[f] \varphi$.

Corollary 5 Every formula with dynamic modalities $[f]$ is logically equivalent to one without.

Note that, depending on the profile updates that are being used, we need increasing expressive power to obtain suitable reduction axioms. In particular, where $f$ is simple (cf. Section 6.3), $[f]$ can be reduced in the languages $\mathfrak{L}$ and $\mathfrak{L}^{[\forall]}$. For finitary $f,[f]$ can be reduced in $\mathfrak{L}_{f}$, resp. $\mathfrak{L}_{f}^{[\forall]}$. Finally, for the class of arbitrary profile updates, we need the full expressive power of $\mathfrak{L}_{\infty}$, resp. $\mathfrak{L}_{\infty}^{[\forall]} \cdot 33$

\section{Summary and Outlook}

In this paper, we have argued that pooling modalities provide a highly expressive and well-behaved extension of the standard multi-index language interpreted over neighbourhood models. We gave a number of foundational results and introduced useful notation and terminology, showing in particular how pooling modalities allow us to axiomatize a variety of dynamic operators.

Given that pooling occurs naturally within a broad variety of application contexts - epistemic, doxastic, deontic, or pertaining to agency - that each come with specific frame conditions, a general method for proving completeness is called for. Such a method is provided in the companion paper [43], combining a canonical model construction with a combinatoric tool, dubbed the puzzle piece construction. In that paper, we also establish and discuss the finite model property as well as compactness for a number of logics with pooling modalities.

We end this paper with a survey of some topics for future work concerning pointwise intersection and pooling modalities.

1. Bisimulation is a standard vehicle for analyzing modal equivalence and expressivity of the modal language. For classical modal logics, similar characterizations of modal equivalence have been investigated in recent work [22]. A natural question, hence, is for a suitable characterization of modal equivalence in languages with pooling modalities (cf. Section 5.1). Relatedly, one may investigate the possibility of a standard translation from $\mathfrak{L}_{\infty}^{[\forall]}$ or one of its sublanguages into first-order logic, again following [22]. Such translations are known to generate numerous important results such as compactness and enumerability.

2. It is well-known that classical modal logics can be embedded into normal modal logics. In particular, monotonic operators can be represented by a combination

\footnotetext{
${ }^{33}$ Relying on examples such as those in Section 5.1, it can be shown that whenever $f(i)$ contains proper pooling profiles $M$ with $|I(M)|>1$ or with $M(j)>1$ for some $j \in I$, a formula such as $[f] \square_{i} p$ cannot be expressed in $\mathfrak{L}^{[\forall]}$. As a corollary, there can be no reduction axioms for such $[f]$ in $\mathfrak{L}^{[\forall]}$. Likewise, whenever $f(i)$ contains infinitary pooling profiles, then $[f] \square_{i}$ cannot be expressed in $\mathfrak{L}_{f}^{[\forall]}$.
} 
of two normal modal operators; for the embedding of arbitrary classical modal operators, three normal modalities are required [25]. Similarly, one may ask whether logics featuring pooling modalities can be embedded in normal modal logics, using intersection modalities. While this has been done for the specific case of coalition logic (which is a fragment of group STIT, cf. [7]), a more general recipe for such embeddings is still lacking.

3. Dynamic operations such as the ones discussed in Section 6 have formal connections to products of neighborhood systems in a similar way that product models of dynamic epistemic logic can be seen as a special type of product model [46]. Also here, the exact relationship remains to be worked out.

4. Finally, as mentioned in the introduction, pointwise intersection is just one prototypical example of combinatorics over neighbourhood sets. Such operations form a larger family that comprises, for instance, neighbourhood operations motivated by PDL's non-deterministic choice or sequential composition [33, 38]. It remains to be seen how the languages and logics introduced in $[33,38]$ combine with pooling modalities. Relatedly, one may also generalize pointwise intersection to other boolean operations of union and negation, and investigate the expressivity and logical properties of ensuing systems.

Acknowledgements We are greatly indebted to two anonymous referees for their incisive comments on earlier versions of this paper. Frederik Van De Putte's research was funded by a Marie SkłodowskaCurie Fellowship (grant agreement ID: 795329), by a grant from the Research Foundation - Flanders (FWO-Vlaanderen), no. 12Q1918N, and by a grant from the Dutch Research Council (NWO), no. VI.Vidi.191.105. The work of Dominik Klein was partially supported by the Deutsche Forschungsgemeinschaft (DFG) and Agence Nationale de la Recherche (ANR) as part of the joint project Collective Attitude Formation [RO 4548/8-1], by DFG and Grantová Agentura České Republiky (GAČR) through the joint project From Shared Evidence to Group Attitudes [RO 4548/6-1], by DFG through the network grants Simulations of Social Scientific Inquiry [426833574] and Foundations, Applications and Theory of Inductive Logic [432308570], and by the National Science Foundation of China as part of the project Logics of Information Flow in Social Networks [17ZDA026].

Open Access This article is licensed under a Creative Commons Attribution 4.0 International License, which permits use, sharing, adaptation, distribution and reproduction in any medium or format, as long as you give appropriate credit to the original author(s) and the source, provide a link to the Creative Commons licence, and indicate if changes were made. The images or other third party material in this article are included in the article's Creative Commons licence, unless indicated otherwise in a credit line to the material. If material is not included in the article's Creative Commons licence and your intended use is not permitted by statutory regulation or exceeds the permitted use, you will need to obtain permission directly from the copyright holder. To view a copy of this licence, visit http://creativecommons.org/licenses/by/4.0/.

\section{References}

1. Ågotnes, T., \& Wáng, Y. N. (2017). Resolving distributed knowledge. Artificial Intelligence, 252, $1-21$.

2. Areces, C., \& Figueira, D. (2009). Which semantics for neighbourhood semantics? In Proceedings of the 21st International Jont Conference on Artifical Intelligence, IJCAI' (pp. 671-676). San Francisco: Morgan Kaufmann Publishers Inc.

3. Baltag, A., Bezhanishvili, N., Özgün, A., \& Smets, S. (2016). Justified belief and the topology of evidence. In J. Väänänen, Å. Hirvonen, \& R. de Queiroz (Eds.) Logic, Language, Information, and Computation (pp. 83-103). Berlin: Springer. 
4. Baltag, A., Moss, L. S., \& Solecki, S. (1998). The logic of public announcements, common knowledge, and private suspicions. In Proceedings of the 7th conference on Theoretical aspects of rationality and knowledge (pp. 43-56).

5. Blum, C., \& Zuber, C. I. (2016). Liquid democracy: Potentials, problems, and perspectives. Journal of Political Philosophy, 24(2), 162-182.

6. Brill, M. (2018). Interactive democracy. In Proceedings of the 17th International Conference on Autonomous Agents and MultiAgent Systems, AAMAS '18 (pp. 1183-1187). Richland: International Foundation for Autonomous Agents and Multiagent Systems.

7. Broersen, J., Herzig, A., \& Troquard, N. (2007). A normal simulation of coalition logic and an epistemic extension. In D. Samet (Ed.) Proceedings of the 11th Conference on Theoretical Aspects of Rationality and Knowledge, TARK'07 (pp. 92-101). New York: ACM.

8. Brown, M. A. (1988). On the logic of ability. Journal of Philosophical Logic, 17(1), 1-26.

9. José, M., Carmo, C. L. M., \& Jones, A. J. I. (2002). Deontic logic and contrary-to-duties. In D. M. Gabay, \& F. Guenther (Eds.) Handbook of Philosophical Logic. 2nd edn., (Vol. 8 pp. 147-264). Kluwer Academic Publishers.

10. Chellas, B. (1980). Modal Logic: An Introduction. Cambridge: Cambridge university press.

11. Christoff, Z., \& Grossi, D. (2017). Binary voting with delegable proxy: An analysis of liquid democracy. In J. Lang (Ed.) Proceedings Sixteenth Conference on Theoretical Aspects of Rationality and Knowledge, volume 251 of Electronic Proceedings in Theoretical Computer Science (pp. 134-150). Open Publishing Association.

12. Elgesem, D. (1997). The modal logic of agency. Nordic Journal of Philosophical Logic, 2, 1-46.

13. Fagin, R., Halpern, J. Y., Moses, Y., \& Vardi, M. Y. (2003). Reasoning about Knowledge. Cambridge: MIT press.

14. Gerbrandy, J. (1999). Bisimulations on Planet Kripke: Phd thesis University of Amsterdam.

15. Goble, J. (2000). Multiplex semantics for deontic logic. Nordic Journal of Philosophical Logic, 5, 113-134.

16. Goble, L. (2004). Preference semantics for deontic logic. Part II: Multiplex models. Logique et Analyse, 185-188, 335-363.

17. Goble, L. (2005). A logic for deontic dilemmas. Journal of Applied Logic, 3, 461-483.

18. Goranko, V., \& Passy, S. (1992). Using the universal modality Gains and questions. Journal of Logic and Computation, 2(1), 5-30.

19. Governatori, G., \& Rotolo, A. (2005). On the axiomatisation of elgesem's logic of agency and ability. Journal of Philosophical Logic, 34(4), 403-431.

20. Hansen, H. H., \& Kupke, C. (2015). Weak completeness of coalgebraic dynamic logics. In R. Matthes, \& M. Mio (Eds.) Proceedings Tenth International Workshop on Fixed Points in Computer Science, volume 191 of Electronic Proceedings in Theoretical Computer Science (pp. 90-104). Berlin: Open Publishing Association.

21. Hansen, H. H., Kupke, C., \& Leal, R. A. (2014). Strong completeness for iteration-free coalgebraic dynamic logics. In J. Diaz, I. Lanese, \& D. Sangiorgi (Eds.) Theoretical Computer Science (pp. 281295). Berlin: Springer.

22. Hansen, H. H., Kupke, C., \& Pacuit, E. (2009). Neighbourhood structures: Bisimilarity and basic model theory. Logical Methods in Computer Science, 5(2).

23. Harel, D., Kozen, D., \& Tiuryn, J. (2000). Dynamic Logic. Cambridge: MIT press.

24. Klein, D. H., \& Marra, A. (2020). From oughts to goals: A logic for enkrasia. Studia Logica, 108, $85-128$.

25. Kracht, M., \& Wolter, F. (1999). Normal monomodal logics can simulate all others. The Journal of Symbolic Logic, 64(1), 99-138.

26. Lewis, D. (1973). Counterfactuals. Cambridge: Harvard University Press.

27. McKinsey, J. C. C. ., \& Tarski, A. (1944). The algebra of topology. Annals of Mathematics, 45, 141191.

28. McNamara, P. (2018). Toward a systematization of logics for monadic and dyadic agency \& ability (preliminary version). In Proceedings for Deontic Logic and Normative Systems (Deon 2018). Utrecht: College Publications.

29. Montague, R. (1970). Universal grammar. Theoria, 36, 373-398.

30. Moss, R., \& Parikh, R. (1992). Topological reasoning and the logic of knowledge. In Y. Moses (Ed.) Proceedings of TARK IV. Morgan Kaufmann. 
31. Pacuit, E. (2017). Neighbourhood semantics for modal logic: Springer.

32. Pauly, M. (2002). A modal logic for coalitional power in games. Journal of Logic and Computation, 1(1), 149-166.

33. Pauly, M., \& Parikh, R. (2003). Game logic - an overview. Studia Logica, 75, 165-182.

34. Roelofsen, F. (2007). Distributed knowledge. Journal of Applied Non-Classical Logics, 17(2), 255273.

35. Scott, D. (1970). Advice on Modal Logic, (pp. 143-173). Netherlands: Springer.

36. Segerberg, K. (1971). An essay in classical modal logic.

37. van Benthem, J., Bezhanishvili, N., \& Enqvist, S. (2019). A new game equivalence, its logic and algebra. Journal of Philosophical Logic, 48(4), 649-684.

38. van Benthem, J., Bezhanishvili, N., \& Enqvist, S. (2019). A propositional dynamic logic for instantial neighborhood semantics. Studia Logica, 107, 719-751.

39. van Benthem, J., \& Bezhanisvilli, G. (2007). Modal logics of space. In Handbook of Spatial Logic (pp. 217-298). Springer.

40. van Benthem, J., \& Pacuit, E. (2011). Dynamic logics of evidence-based beliefs. Studia Logica, 99(1), 61.

41. Van De Putte, F. (2017). That will do: Logics of deontic necessity and sufficiency. Erkenntnis, 82(3), 473-511.

42. Van De Putte, F., \& Klein, D. (2018). Pointwise intersection in neighbourhood modal logic. In G. Bezhanishvili, \& G. D’Agostino (Eds.) Advances in Modal Logic (AiML 12) (pp. 591-610). College Publications.

43. Van De Putte, F., \& Klein, D. (2021). Pooling modalities and pointwise intersection: Axiomatization and decidability. Studia Logica, 109, 47-93.

44. van der HOek, W., van Linder, B., \& Meyer, J.-J. (1999). Group knowledge is not always distributed (neither is it always implicit). Mathematical social sciences, 38, 215-240.

45. van der Hoek, W., \& Wooldridge, M. (2005). On the dynamics of delegation, cooperation, and control: A logical account. In Proceedings of the Fourth International Joint Conference on Autonomous Agents and Multiagent Systems, AAMAS '05 (p. 701708). New York: Association for Computing Machinery.

46. van Ditmarsch, H., van Der Hoek, W., \& Kooi, B. (2007). Dynamic epistemic logic, volume 337 of Synthese Library. Springer.

Publisher's Note Springer Nature remains neutral with regard to jurisdictional claims in published maps and institutional affiliations. 